\title{
TENDENCIA Y FACTORES ASOCIADOS A LA FRECUENCIA DE CEPILLADO DENTAL EN MENORES DE DOCE AÑOS, PERÚ 2013-2018
}

\author{
Gilmer Solis ${ }^{1, a}$, Eraldo Pesaressi²,b, Wilfredo Mormontoy ${ }^{1, c}$
}

\begin{abstract}
RESUMEN
Objetivos. Determinar la tendencia y factores asociados al cepillado de dos o más veces por día en niños menores de doce años entre el 2013 y 2018 en Perú. Materiales y métodos. Se realizó un estudio transversal mediante análisis secundario de datos reportados por la Encuesta Demográfica y de Salud Familiar. Se consideró la información de niños con al menos un cepillado al día, considerando como «Adecuada Frecuencia de Cepillado Dental» (AFC) para quienes reportaban de dos a más; se generaron medidas porcentuales por ámbito geográfico, características sanitarias, de vivienda y hogar. Se determinaron los factores asociados a la AFC mediante regresión logística binaria para encuestas complejas, la variabilidad de la tendencia se identificó mediante regresión joinpoint con un 95\% de confianza. Resultados. En el 2013, la AFC fue del $79,0 \%$ con un aumento a $83,9 \%$ para el 2018 ; se identificó que cuatro de 25 espacios geográficos se mantuvieron sin variación de AFC, y otros cuatro disminuyeron. Todas las características sanitarias mostraron favorecer el cepillado, destacándose que el no compartir el cepillo generó un OR de 2,30, IC 95\%: 1,46 a 3,60. La región natural, el tipo de lugar de residencia y el quintil de riqueza afectaron la AFC; la AFC fue mayor según se incrementa el grupo etario. Conclusiones. El porcentaje de AFC ha aumentado en los últimos años, siendo influenciado favorablemente por los aspectos sanitarios; a nivel rural este porcentaje es significativamente menor, algo que también se evidencia en la región sierra.
\end{abstract}

Palabras clave: Cepillado Dental; Encuesta de Salud; Factores Epidemiológicos; Higiene Bucal. (Fuente: DeCS BIREME).

\section{TREND AND FACTORS ASSOCIATED WITH THE FREQUENCY OF TOOTH BRUSHING IN CHILDREN UNDER TWELVE YEARS OLD, PERU 2013-2018}

\begin{abstract}
Objetivos. To determine the trend and factors associated with brushing two or more times per day in children under twelve years of age between 2013 and 2018 in Peru. Materials and Methods. A cross-sectional study was conducted by secondary analysis of data reported by the Demographic and Family Health Survey (ENDES). Information on children with at least one brushing per day was taken into account, considering as an "Adequate Toothbrushing Frequency" (ATF) for those who reported brushing two to more times per day. Percentage measures were generated by geographical area, health, housing, and household characteristics. Factors associated with an ATF were determined by using binary logistic regression for complex surveys, trend variability was identified by joinpoint regression with a $95 \%$ confidence. Results. In 2013 , the ATF was $79.0 \%$, increasing to $83.9 \%$ by 2018 . Four out of 25 geographic areas were identified as having no variation on the ATF, and four others decreased. All sanitary characteristics showed favoring brushing, emphasizing that not sharing the toothbrush generated a $2.30 \mathrm{OR}, 95 \% \mathrm{Cl}: 1.46$ to 3.60 . The natural region, type of place of residence, and wealth index quintile affected the ATF. The ATF was higher as the age group increased. Conclusions. The percentage of ATF has increased in recent years, being favorably influenced by health aspects; in rural areas, this percentage is significantly lower, something that is also evident in the Highland region.
\end{abstract}

Keywords: Toothbrushing, Health Surveys, Epidemiologic Factors, Oral Hygiene. (Source: MeSH NLM).

\section{INTRODUCCIÓN}

La salud bucal es primordial para el bienestar general, en la que una dentición sana y funcional durante toda la vida, permitirá funciones humanas esenciales como la masticación, sonreír, hablar y socializar ${ }^{(1)}$. La caries dental y enfermedad periodontal son condiciones de etiología multifactorial, consideradas problemas de salud pública global ${ }^{(2,3)}$, ya que lideran el ranking de las enfermedades más prevalentes ${ }^{(4)}$. Estas condiciones pueden poner en riesgo el desarrollo integral de un individuo limitándolo en sus funciones básicas y afectando severamente su

1 Departamento de Estadística, Demografía, Humanidades y Ciencias Sociales, Facultad de Ciencias y Filosofía. Universidad Peruana Cayetano Heredia. Lima, Perú.

2 Departamento de Odontopediatría, Facultad de Odontología. Universidad San Martin de Porres. Lima, Perú.

a Cirujano Dentista; ${ }^{\text {b }}$ Maestro en Odontología; ${ }^{\mathrm{c}}$ Maestro de Salud Pública.

Recibido: 18/10/2019 Aprobado: 06/11/2019 En línea: 03/12/2019

Citar como: Solis G, Pesaressi E, Mormontoy W. Tendencia y factores asociados a la frecuencia de cepillado dental en menores de doce años, Perú 2013-2018. Rev Peru Med Exp Salud Publica. 2019;36(4):562-72. doi: 10.17843/rpmesp.2019.364.4888. 
calidad de vida ${ }^{(5,6)}$. Sin embargo, ambas enfermedades pueden prevenirse si es que se controlan adecuadamente los factores moduladores: dieta, visitas regulares al odontólogo y la desorganización del biofilm mediante el cepillado dental ${ }^{(7,8)}$.

El cepillado debe realizarse higienizando todas las superficies dentales, lengua y encías con una frecuencia de al menos dos veces por día, una de las cuales se sugiere sea antes de dormir o idealmente 30 minutos después de cada comida ${ }^{(9)}$; tras estos períodos críticos los ácidos producidos por el metabolismo del biofilm pueden acelerar la desmineralización dental generando daño micro-estructural que posteriormente podría manifestarse como una lesión clínicamente visible ${ }^{(3)}$. Se debe considerar además que la regularidad de las visitas al odontólogo basadas en el riesgo individual de cada paciente, aumentan la probabilidad de detección de signos patológicos iniciales; complementariamente, hábitos protectores como un cepillado dental efectivo y frecuente, uso de hilo dental y colutorios, una dieta balanceada están asociadas con un menor riesgo a incidencia de caries dental y enfermedad periodontal ${ }^{(7,10,11)}$.

La higiene bucal inadecuada es un factor predictivo de enfermedad periodontal, incrementando el riesgo entre dos a cinco veces, cuando se les compara con individuos con buena higiene ${ }^{(12)}$; así también, la pérdida dental y la presencia de patógenos orales se han relacionado con enfermedades sistémicas no transmisibles como diabetes, neumonía y enfermedades circulatorias ${ }^{(13-15)}$. Los programas preventivos en salud bucal han quedado relegados a iniciativas aisladas en comparación a las de salud general; sin embargo, promover hábitos saludables desde edades tempranas puede ser una medida costoefectiva, considerando que las enfermedades bucales en estadios avanzados son el cuarto problema de salud más costoso de tratar ${ }^{(1,16)}$.

Para poder realizar medidas de promoción de la salud mediante el cepillado dental efectivo, es necesario conocer las tendencias de la población peruana, especialmente en grupos vulnerables. El objetivo del presente trabajo fue estimar frecuencia de cepillado de dos o más veces al día de niños peruanos menores de 12 años, valorando tendencias entre el 2013 y 2018; y determinar los factores asociados.

\section{MATERIALES Y MÉTODOS}

\section{DISEÑO Y POBLACIÓN DE ESTUDIO}

El estudio fue observacional y analítico enmarcado en el diseño de corte transversal de medidas repetidas a nivel poblacional ${ }^{(17)}$. Se realizó un análisis de fuentes secundarias con las bases de datos de la Encuesta

\section{MENSAJES CLAVE}

Motivación para realizar el estudio. En el Perú existen estudios enfocados en subpoblaciones, las cuales no llegan a ser representativas de la realidad nacional, por lo que se desconoce el panorama situacional sobre la higiene bucal actual y sus cambios en el tiempo.

Principales hallazgos. A lo largo de los años existe un incremento monótono en el porcentaje de niños con adecuada frecuencia de cepillado dental, con variaciones importantes según regiones y algunas características sanitarias, de vivienda y hogar.

Implicancias. La identificación específica de grupos que requieren mayor atención, ayudaría a implementar estrategias sanitarias específicas, que garanticen mejorar la higiene y subsecuentemente la salud bucal.

Demográfica y de Salud Familiar (ENDES) realizados a nivel de todo el Perú entre los años 2013 al 2018 por el Instituto Nacional de Estadística e Informática (INEI) las cuales se encuentran disponibles de manera pública (http:// iinei.inei.gob.pe/microdatos/). La unidad de análisis de la ENDES son residentes habituales de viviendas particulares a nivel nacional, quienes hayan pernoctado en el domicilio seleccionado la noche anterior a la encuesta, siguiendo un muestreo probabilístico, estratificado y bietápico (Conglomerados y Viviendas) ${ }^{(18,19)}$.

Se empleó la información de la sección «Salud Bucal» del Cuestionario de Salud, donde se encuentran los registros de niños menores de 12 años. Para obtener las variables adicionales fue necesario fusionar los archivos del Cuestionario de Hogar sobre las secciones «Datos Básicos de los hogares» y «Miembros del Hogar», así también la sección «Características de Hogar y Vivienda»; este proceso se llevó a cabo por cada año encuestado de forma separada, para posteriormente ser condensadas en una única base.

\section{VARIABLES}

La variable dependiente fue la frecuencia de cepillado dental, la cual tomó valores dicotomizados (una vez al día y dos a más veces al día), se generó tras la recodificación de la variable QS811 ("Cuantas veces al día») referida a cepillado dental, la cual originalmente proviene del Cuestionario de Salud; este dato es reportado por la madre, el niño u otro adulto en el hogar bajo los siguientes valores: una vez al día, dos veces al día, tres veces al día y cuatro o más veces al día. Se consideró como «Adecuada Frecuencia de Cepillado Dental» (AFC) a aquellos niños quienes se encontraban en la categoría «2 a más veces al día» de la variable dependiente, se identificó esta característica según ámbito geográfico mediante la variable «Identificación geográfica» (24 regiones y la provincia constitucional del callao). 
Se seleccionaron como variables independientes aquellas que pudieran estar vinculadas a la AFC, agrupándose en tres categorías: Características sanitarias, de la vivienda, y del hogar. Se consideraron como características sanitarias, a todas aquellas variables que expresan información de la conducta de higiene y de los servicios de salud, las características de la vivienda fueron todas aquellas que corresponden a la ubicación geográfica y acceso a servicios, los aspectos que comprenden las características del hogar estuvieron orientadas a la organización funcional y aspectos de sus miembros; en el material suplementario (Anexo 1) se presentan las variables incluidas según lo definido por la ENDES, así como las nuevas etiquetas y valores asignados para el estudio.

Entre las características del hogar, la variable edad del niño proveniente de la base de salud, se categorizó según criterio de aplicación clínica en: 1 a 2 años (infantes), 3 a 5 años (preescolares con dentición decidua), 6 a 8 años (escolares con dentición mixta que requieren ser cepillados por sus padres), y de 9 a 11 años (púberes con dentición mixta que no requieren ser cepillado por sus padres). De forma complementaria, se utilizaron las variables «Número de miembros en el hogar» y «Número de cuartos» para calcular el Índice de Hacinamiento (Sin hacinamiento, con hacinamiento), considerándose con hacinamiento a hogares en las que habitan de tres a más personas por habitación ${ }^{(20)}$. Adicionalmente al tratarse de una investigación de tendencia, se consideró una cuarta característica referida al diseño del estudio proveniente de la variable año de encuesta.

\section{ANÁLISIS ESTADÍSTICO}

El análisis de los datos fue ejecutado empleando el paquete estadístico Stata v14.2 (Stata Corporation, College Station, Texas, USA) considerando el diseño muestral complejo respetando la estratificación y la identificación de conglomerados de la ENDES, además se empleó el factor de ponderación para niños menores de 12 años consignado en la base de datos del cuestionario de salud; toda esta preparación se llevó a cabo empleando el comando svy.

Se identificó el porcentaje e intervalo de confianza (IC) al 95\% para la AFC en niños menores de 12 años, empleándose el test Chi Cuadrado de Pearson para valorar las diferencias significativas según características sanitarias, de vivienda y hogar dentro de cada año evaluado; se empleó un modelamiento lineal generalizado (GLM) con opción de familia binomial y link identity para establecer la diferencia e IC al 95\% entre el porcentaje de AFC del 2013 y 2018. Se estableció una diferencia corregida (relativa) de dicha variación al ajustarla por el valor del 2013 (diferencia 20182013/valor del 2013 x 100).

Se evaluó la correlación entre la AFC en cada categoría con el año evaluado mediante la prueba coeficiente de correlación de Pearson o la de Spearman-Brown (previa identificación de normalidad mediante test ShapiroWilk) a fin de establecer si había direccionalidad en los cambios de la estimación porcentual del AFC a lo largo de los años. Para establecer de forma multivariada los factores que modificaban la AFC se realizó una regresión logística binaria ${ }^{(21)}$, ajustada a todas las variables independientes planteadas, según las características previamente definidas; empleando para dicho fin el registro de 140580 sujetos que presentaron datos completos para las características de interés. Es importante señalar que en el modelo multivariado no se consideró a la variable «Hogar con Bomba de Agua» (que valora el impacto del acceso al agua en el hogar como soporte de las medidas de buena higiene), ya que estas forman parte de un total de 113 variables que sirven para establecer el Quintil de Riqueza, las cuales de mantenerse generarían colinealidad e inflación de la varianza.

La variabilidad en el comportamiento de la tendencia de la AFC a través de los años (2013 al 2018) se estableció con ayuda del software Joinpoint Desktop de la versión 4.7.0,0 (Division of Cancer Control and Population Sciences, National Cancer Institute), aplicando una regresión joinpoint de porcentajes con el que se identificó el Porcentaje de Cambio Anual (APC: Annual Percent Change) ${ }^{(22,23)}$. Todas las pruebas inferenciales, tanto bivariadas como multivariadas se trabajaron con un nivel de significancia estadística de 0,05.

\section{CONSIDERACIONES ÉTICAS}

El presente estudio, al enfocarse en la evaluación de una base de datos secundaria, no requirió aprobación previa por un comité de ética; toda vez que la información se encontraba disponible de manera pública a través del portal del INEI.

\section{RESULTADOS}

Del registro de 140589 menores de 12 años, 3742 (2,7\%) corresponden al 2013, $17596(12,5 \%)$ al 2014, 31632 $(22,5 \%)$ al 2015, para el 2016 se evaluaron a 28811 (20,5\%), en el 2017 a $28091(20,0 \%)$, y finalmente a $30717(21,8 \%)$ en el 2018 . Las edades presentaron una media general de $6,1 \pm 3,0$, cuyos valores por año fueron de 6,7 $\pm 2,8$ para el 2013 , el 2014 se encontró un valor de 6,7 $\pm 2,9$, para el 2015 de $6,0 \pm 3,0$, en el 2016 fue también de 6,0 $\pm 3,0$, mientras que para el 2017 y 2018 de $6,0 \pm 3,0$ y $5,9 \pm 3,0$ respectivamente.

En la evaluación de AFC por ámbito geográfico (Tabla 1) se evidencia variabilidad en las proporciones entre regiones $(p<0,001)$ para cada año evaluado, la AFC no eran las mismas a lo largo de los años siendo que 
Tabla 1. Distribución de la adecuada frecuencia de cepillado dental en menores de 12 años según ámbito geográfico, Perú 2013-2018

\begin{tabular}{|c|c|c|c|c|c|c|c|c|c|c|}
\hline \multirow[b]{2}{*}{$\begin{array}{l}\text { Ámbito } \\
\text { geográfico }\end{array}$} & 2013 & 2014 & 2015 & 2016 & 2017 & 2018 & \multirow[b]{2}{*}{$\begin{array}{c}\text { Diferencia } \\
2013-2018 \\
\%(\text { IC } 95 \%)^{*}\end{array}$} & \multirow[b]{2}{*}{$\begin{array}{l}\text { Valor p de la } \\
\text { Diferencia } \\
2013 \text { - 2018* }\end{array}$} & \multirow[b]{2}{*}{$\begin{array}{c}\text { Coeficiente } \\
\text { de } \\
\text { Correlación }^{\star *}\end{array}$} & \multirow[b]{2}{*}{$\begin{array}{l}\text { Valor } \mathbf{p} \text { de la } \\
\text { Correlación** }\end{array}$} \\
\hline & $\begin{array}{c}\% \\
\text { (IC 95\%) }\end{array}$ & $\begin{array}{c}\% \\
\text { (IC 95\%) }\end{array}$ & $\begin{array}{c}\% \\
\text { (IC 95\%) }\end{array}$ & $\begin{array}{c}\% \\
\text { (IC 95\%) }\end{array}$ & $\begin{array}{c}\% \\
\text { (IC 95\%) }\end{array}$ & $\begin{array}{c}\% \\
\text { (IC } 95 \%)\end{array}$ & & & & \\
\hline Amazonas & $\begin{array}{c}87,1 \\
(78,3 \text { a } 92,7)\end{array}$ & $\begin{array}{c}84,6 \\
(80,2 \text { a } 88,1)\end{array}$ & $\begin{array}{c}86,9 \\
(84,1 \text { a } 89,2)\end{array}$ & $\begin{array}{c}85,0 \\
(81,4 \text { a } 88,0)\end{array}$ & $\begin{array}{c}86,2 \\
(83,9 \text { a } 88,3)\end{array}$ & $\begin{array}{c}86,0 \\
(83,5 \text { a } 88,2)\end{array}$ & $\begin{array}{c}-1,1 \\
(-8,5 \text { a } 6,3)\end{array}$ & 0,761 & $-0,139^{\dagger}$ & 0,793 \\
\hline Ancash & $\begin{array}{c}80,9 \\
(71,8 \text { a } 87,6)\end{array}$ & $\begin{array}{c}83,9 \\
(79,0 \text { a } 87,8)\end{array}$ & $\begin{array}{c}83,0 \\
(78,2 \text { a } 87,0)\end{array}$ & $\begin{array}{c}85,2 \\
(81,9 \text { a } 88,0)\end{array}$ & $\begin{array}{c}85,3 \\
(81,6 \text { a } 88,3)\end{array}$ & $\begin{array}{c}85,6 \\
(82,8 \text { a } 88,0)\end{array}$ & $\begin{array}{c}4,7 \\
(-3,7 \text { a } 13,0)\end{array}$ & 0,269 & $0,885^{\dagger}$ & 0,019 \\
\hline Apurímac & $\begin{array}{c}75,8 \\
(66,4 \text { a } 83,3)\end{array}$ & $\begin{array}{c}78,8 \\
(73,8 \text { a } 83,1)\end{array}$ & $\begin{array}{c}84,0 \\
(79,4 \text { a } 87,7)\end{array}$ & $\begin{array}{c}82,4 \\
(78,3 \text { a } 85,9)\end{array}$ & $\begin{array}{c}80,2 \\
(75,0 \text { a } 84,6)\end{array}$ & $\begin{array}{c}82,2 \\
(79,0 \text { a } 84,9)\end{array}$ & $\begin{array}{c}6,4 \\
(-2,7 \text { a } 15,4)\end{array}$ & 0,161 & $0,625^{\dagger}$ & 0,185 \\
\hline Arequipa & $\begin{array}{c}74,0 \\
(63,3 \text { a } 82,4)\end{array}$ & $\begin{array}{c}71,5 \\
(65,7 \text { a } 76,7)\end{array}$ & $\begin{array}{c}72,7 \\
(68,9 ; 76,2)\end{array}$ & $\begin{array}{c}77,1 \\
(73,4 \text { a } 80,5)\end{array}$ & $\begin{array}{c}79,5 \\
(75,8 \text { a } 82,8)\end{array}$ & $\begin{array}{c}78,5 \\
(74,4 \text { a } 82,2)\end{array}$ & $\begin{array}{c}4,5 \\
(-5,9 \text { a } 15,0)\end{array}$ & 0,385 & $0,831^{\dagger}$ & 0,041 \\
\hline Ayacucho & $\begin{array}{c}72,2 \\
(65,6 \text { a } 78,0)\end{array}$ & $\begin{array}{c}75,0 \\
(70,2 \text { a } 79,3)\end{array}$ & $\begin{array}{c}81,4 \\
(78,1 \text { a } 84,4)\end{array}$ & $\begin{array}{c}82,1 \\
(78,7 \text { a } 85,1)\end{array}$ & $\begin{array}{c}85,7 \\
(82,5 \text { a } 88,3)\end{array}$ & $\begin{array}{c}83,5 \\
(80,4 \text { a } 86,1)\end{array}$ & $\begin{array}{c}11,3 \\
(4,4 \text { a } 18,1)\end{array}$ & 0,001 & $0,912^{\dagger}$ & 0,011 \\
\hline Callao & $\begin{array}{c}85,4 \\
(70,8 \text { a } 93,3)\end{array}$ & $\begin{array}{c}85,8 \\
(82,0 \text { a } 88,9)\end{array}$ & $\begin{array}{c}87,0 \\
(84,3 \text { a } 89,2)\end{array}$ & $\begin{array}{c}85,9 \\
(82,8 \text { a } 88,5)\end{array}$ & $\begin{array}{c}85,6 \\
(82,6 \text { a } 88,1)\end{array}$ & $\begin{array}{c}87,2 \\
(84,8 \text { a } 89,3)\end{array}$ & $\begin{array}{c}1,9 \\
(-9,4 \text { a } 13,1)\end{array}$ & 0,732 & $0,515^{\dagger}$ & 0,296 \\
\hline Cusco & $\begin{array}{c}55,0 \\
(42,8 \text { a } 66,6)\end{array}$ & $\begin{array}{c}64,4 \\
(59,1 \text { a } 69,3)\end{array}$ & $\begin{array}{c}76,5 \\
(72,2 \text { a } 80,3)\end{array}$ & $\begin{array}{c}75,3 \\
(70,3 \text { a } 79,6)\end{array}$ & $\begin{array}{c}77,0 \\
(73,0 \text { a } 80,6)\end{array}$ & $\begin{array}{c}74,6 \\
(70,2 \text { a } 78,4)\end{array}$ & $\begin{array}{c}19,5 \\
(6,6 \text { a } 32,4)\end{array}$ & 0,002 & $0,600^{\ddagger}$ & 0,208 \\
\hline Huancavelica & $\begin{array}{c}70,0 \\
(54,7 \text { a } 81,9)\end{array}$ & $\begin{array}{c}71,9 \\
(65,8 \text { a } 77,3)\end{array}$ & $\begin{array}{c}75,6 \\
(70,7 \text { a } 79,9)\end{array}$ & $\begin{array}{c}72,5 \\
(65,0 \text { a } 79,0)\end{array}$ & $\begin{array}{c}66,0 \\
(59,6 \text { a } 71,8)\end{array}$ & $\begin{array}{c}77,2 \\
(73,2 \text { a } 80,8)\end{array}$ & $\begin{array}{c}7,2 \\
(-7,4 \text { a } 21,7)\end{array}$ & 0,347 & $0,203^{\dagger}$ & 0,700 \\
\hline Huánuco & $\begin{array}{c}84,9 \\
(77,2 \text { a } 90,4)\end{array}$ & $\begin{array}{c}75,7 \\
(70,7 \text { a } 80,1)\end{array}$ & $\begin{array}{c}83,9 \\
(80,6 \text { a } 86,7)\end{array}$ & $\begin{array}{c}81,8 \\
(78,7 \text { a } 84,5)\end{array}$ & $\begin{array}{c}81,1 \\
(78,0 \text { a } 83,8)\end{array}$ & $\begin{array}{c}84,9 \\
(82,3 \text { a } 87,1)\end{array}$ & $\begin{array}{c}0,0 \\
(-7,0 \text { a } 7,0)\end{array}$ & 0,992 & $0,058^{\ddagger}$ & 0,913 \\
\hline Ica & $\begin{array}{c}86,4 \\
(76,8 \text { a } 92,4)\end{array}$ & $\begin{array}{c}84,4 \\
(80,9 \text { a } 87,3)\end{array}$ & $\begin{array}{c}87,4 \\
(84,5 \text { a } 89,9)\end{array}$ & $\begin{array}{c}84,4 \\
(81,2 \text { a } 87,1)\end{array}$ & $\begin{array}{c}87,1 \\
(84,8 \text { a } 89,1)\end{array}$ & $\begin{array}{c}87,2 \\
(84,8 \text { a } 89,3)\end{array}$ & $\begin{array}{c}0,8 \\
(-7,2 \text { a } 8,9)\end{array}$ & 0,833 & $0,348^{\dagger}$ & 0,499 \\
\hline Junín & $\begin{array}{c}79,2 \\
(73,3 \text { a } 84,0)\end{array}$ & $\begin{array}{c}73,8 \\
(67,2 \text { a } 79,4)\end{array}$ & $\begin{array}{c}76,1 \\
(71,6 \text { a } 80,1)\end{array}$ & $\begin{array}{c}74,0 \\
(69,3 \text { a } 78,1)\end{array}$ & $\begin{array}{c}79,2 \\
(74,8 \text { a } 83,0)\end{array}$ & $\begin{array}{c}79,2 \\
(75,6 \text { a } 82,4)\end{array}$ & $\begin{array}{c}0,0 \\
(-6,3 \text { a } 6,4)\end{array}$ & 0,991 & $0,287^{\dagger}$ & 0,582 \\
\hline La Libertad & $\begin{array}{c}81,9 \\
(74,1 \text { a } 87,7)\end{array}$ & $\begin{array}{c}79,7 \\
(73,4 \text { a } 84,8)\end{array}$ & $\begin{array}{c}83,0 \\
(79,7 \text { a } 85,9)\end{array}$ & $\begin{array}{c}86,8 \\
(83,9 \text { a 89,3) }\end{array}$ & $\begin{array}{c}88,5 \\
(85,8 \text { a } 90,7)\end{array}$ & $\begin{array}{c}87,3 \\
(84,8 \text { a } 89,4)\end{array}$ & $\begin{array}{c}5,4 \\
(-1,8 \text { a } 12,6)\end{array}$ & 0,155 & $0,874^{\dagger}$ & 0,023 \\
\hline Lima & $\begin{array}{c}81,0 \\
(73,5 \text { a } 86,7)\end{array}$ & $\begin{array}{c}85,3 \\
(82,9 \text { a } 87,3)\end{array}$ & $\begin{array}{c}84,9 \\
(83,2 \text { a } 86,4)\end{array}$ & $\begin{array}{c}86,0 \\
(84,3 ; 87,6)\end{array}$ & $\begin{array}{c}86,4 \\
(84,8 \text { a } 87,9)\end{array}$ & $\begin{array}{c}85,8 \\
(84,3 \text { a } 87,1)\end{array}$ & $\begin{array}{c}4,8 \\
(-2,0 \text { a } 11,6)\end{array}$ & 0,163 & $0,771^{\ddagger}$ & 0,072 \\
\hline Loreto & $\begin{array}{c}82,8 \\
(77,9 \text { a } 86,8)\end{array}$ & $\begin{array}{c}79,2 \\
(75,1 \text { a } 82,7)\end{array}$ & $\begin{array}{c}82,1 \\
(77,8 \text { a } 85,7)\end{array}$ & $\begin{array}{c}82,5 \\
(78,3 \text { a } 85,9)\end{array}$ & $\begin{array}{c}84,7 \\
(81,9 \text { a } 87,2)\end{array}$ & $\begin{array}{c}87,3 \\
(85,2 \text { a } 89,1)\end{array}$ & $\begin{array}{c}4,4 \\
(-0,5 \text { a } 9,3)\end{array}$ & 0,068 & $0,776 \dagger$ & 0,070 \\
\hline $\begin{array}{l}\text { Madre de } \\
\text { Dios }\end{array}$ & $\begin{array}{c}83,2 \\
(74,3 \text { a } 89,4)\end{array}$ & $\begin{array}{c}72,9 \\
(68,7 \text { a } 76,7)\end{array}$ & $\begin{array}{c}78,5 \\
(73,8 \text { a 82,6) }\end{array}$ & $\begin{array}{c}77,4 \\
(73,7 \text { a } 80,6)\end{array}$ & $\begin{array}{c}71,9 \\
(67,7 \text { a } 75,8)\end{array}$ & $\begin{array}{c}72,8 \\
(69,2 \text { a } 76,1)\end{array}$ & $\begin{array}{c}-10,4 \\
(-18,7 a-2,1)\end{array}$ & 0,014 & $-0,682 \dagger$ & 0,136 \\
\hline Moquegua & $\begin{array}{c}74,5 \\
(68,6 \text { a } 79,5)\end{array}$ & $\begin{array}{c}79,5 \\
(75,2 \text { a } 83,2)\end{array}$ & $\begin{array}{c}80,4 \\
(76,6 \text { a } 83,7)\end{array}$ & $\begin{array}{c}79,4 \\
(75,9 \text { a } 82,5)\end{array}$ & $\begin{array}{c}74,6 \\
(70,7 \text { a } 78,1)\end{array}$ & $\begin{array}{c}79,7 \\
(75,3 \text { a } 83,5)\end{array}$ & $\begin{array}{c}5,3 \\
(-1,6 \text { a } 12,1)\end{array}$ & 0,133 & $0,314 \ddagger$ & 0,544 \\
\hline Pasco & $\begin{array}{c}69,9 \\
(61,9 \text { a } 76,8)\end{array}$ & $\begin{array}{c}88,6 \\
(85,5 \text { a } 91,0)\end{array}$ & $\begin{array}{c}82,4 \\
(79,0 \text { a } 85,4)\end{array}$ & $\begin{array}{c}86,2 \\
(83,0 \text { a } 88,8)\end{array}$ & $\begin{array}{c}84,0 \\
(80,4 \text { a } 87,1)\end{array}$ & $\begin{array}{c}79,7 \\
(75,8 \text { a } 83,2)\end{array}$ & $\begin{array}{c}9,9 \\
(1,4 \text { a } 18,3)\end{array}$ & 0,023 & $0,317 \dagger$ & 0,541 \\
\hline Piura & $\begin{array}{c}78,7 \\
(72,1 \text { a } 84,2)\end{array}$ & $\begin{array}{c}82,9 \\
(79,0 \text { a } 86,3)\end{array}$ & $\begin{array}{c}82,8 \\
(80,1 \text { a 85,2) }\end{array}$ & $\begin{array}{c}82,0 \\
(78,2 \text { a } 85,3)\end{array}$ & $\begin{array}{c}85,1 \\
(82,3 \text { a } 87,6)\end{array}$ & $\begin{array}{c}85,3 \\
(82,0 \text { a } 88,1)\end{array}$ & $\begin{array}{c}6,6 \\
(-0,2 \text { a } 13,4)\end{array}$ & 0,052 & $0,861^{\dagger}$ & 0,028 \\
\hline Puno & $\begin{array}{c}66,1 \\
(58,1 \text { a } 73,2)\end{array}$ & $\begin{array}{c}62,7 \\
(56,0 \text { a } 68,9)\end{array}$ & $\begin{array}{c}63,6 \\
(58,6 \text { a } 68,3)\end{array}$ & $\begin{array}{c}64,4 \\
(57,8 \text { a } 70,5)\end{array}$ & $\begin{array}{c}65,2 \\
(59,4 \text { a } 70,6)\end{array}$ & $\begin{array}{c}70,4 \\
(65,1 \text { a } 75,3)\end{array}$ & $\begin{array}{c}4,4 \\
(-4,9 \text { a } 13,7)\end{array}$ & 0,364 & $0,585^{\dagger}$ & 0,223 \\
\hline San Martín & $\begin{array}{c}78,4 \\
(72,1 \text { a } 83,6)\end{array}$ & $\begin{array}{c}82,3 \\
(77,4 \text { a } 86,3)\end{array}$ & $\begin{array}{c}83,4 \\
(79,7 \text { a } 86,6)\end{array}$ & $\begin{array}{c}84,8 \\
(82,1 \text { a } 87,2)\end{array}$ & $\begin{array}{c}85,1 \\
(82,4 \text { a } 87,4)\end{array}$ & $\begin{array}{c}84,9 \\
(81,8 \text { a } 87,6)\end{array}$ & $\begin{array}{c}6,5 \\
(0,0 \text { a } 13,0)\end{array}$ & 0,056 & $0,881^{\dagger}$ & 0,020 \\
\hline Tacna & $\begin{array}{c}77,5 \\
(62,6 \text { a } 87,6)\end{array}$ & $\begin{array}{c}77,8 \\
(72,9 \text { a } 82,1)\end{array}$ & $\begin{array}{c}76,5 \\
(72,3 \text { a } 80,3)\end{array}$ & $\begin{array}{c}80,6 \\
(76,5 \text { a } 84,1)\end{array}$ & $\begin{array}{c}77,6 \\
(73,9 \text { a } 81,0)\end{array}$ & $\begin{array}{c}76,7 \\
(72,7 \text { a } 80,2)\end{array}$ & $\begin{array}{c}-0,8 \\
(-14,0 \text { a } 12,4)\end{array}$ & 0,902 & $-0,018^{\dagger}$ & 0,973 \\
\hline Tumbes & $\begin{array}{c}74,8 \\
(65,2 \text { a } 82,4)\end{array}$ & $\begin{array}{c}83,3 \\
(79,2 \text { a } 86,8)\end{array}$ & $\begin{array}{c}83,0 \\
(80,0 \text { a } 85,6)\end{array}$ & $\begin{array}{c}82,4 \\
(79,9 \text { a } 84,6)\end{array}$ & $\begin{array}{c}83,3 \\
(80,6 \text { a } 85,6)\end{array}$ & $\begin{array}{c}80,0 \\
(77,3 \text { a } 82,4)\end{array}$ & $\begin{array}{c}5,2 \\
(-3,9 \text { a } 14,3)\end{array}$ & 0,257 & $0,116^{\ddagger}$ & 0,827 \\
\hline Ucayali & $\begin{array}{c}70,9 \\
(62,5 \text { a } 78,1)\end{array}$ & $\begin{array}{c}76,4 \\
(72,5 \text { a } 80,0)\end{array}$ & $\begin{array}{c}74,3 \\
(70,4 \text { a } 77,9)\end{array}$ & $\begin{array}{c}77,0 \\
(72,5 \text { a } 80,9)\end{array}$ & $\begin{array}{c}76,7 \\
(73,4 \text { a } 79,7)\end{array}$ & $\begin{array}{c}68,1 \\
(65,0 \text { a } 71,1)\end{array}$ & $\begin{array}{c}-2,8 \\
(-11,2 \text { a } 5,7)\end{array}$ & 0,510 & $-0,152^{\dagger}$ & 0,773 \\
\hline Total & $\begin{array}{c}79,0 \\
(76,7 \text { a } 81,2)\end{array}$ & $\begin{array}{c}80,7 \\
(79,6 \text { a } 81,7)\end{array}$ & $\begin{array}{c}82,1 \\
(81,3 \text { a 82,9) }\end{array}$ & $\begin{array}{c}82,0 \\
(81,1 \text { a } 82,9)\end{array}$ & $\begin{array}{c}84,0 \\
(83,3 \text { a } 84,7)\end{array}$ & $\begin{array}{c}83,9 \\
(83,2 \text { a } 84,5)\end{array}$ & $\begin{array}{c}4,8 \\
(2,5 \text { a } 7,2) \\
\end{array}$ & $<0,001$ & $0,959^{\dagger}$ & 0,003 \\
\hline
\end{tabular}

* Diferencia establecida entre los estimadores porcentuales del año 2013 y 2018 mediante regresión logística binaria con link identity.

${ }^{* *}$ Correlación establecida entre los años y el estimador porcentual de 2 a más cepillados por día para cada espacio geográfico.

† Coeficiente de correlación de Pearson.

‡ Coeficiente de correlación de Spearman-Brown. 
el 2013 la región más sobresaliente fue Amazonas $(87,1 \%)$, para el 2014 y 2015 fueron Pasco $(88,6 \%)$ e lca $(87,4 \%)$, La Libertad lideró entre el 2016 al 2018 (86,8\%, $88,5 \%$ y $87,3 \%$ respectivamente). El menor desempeño de higiene para el 2013 fue de Cusco con un 55,0\%, entre el 2014 y el 2017 lo presentó Puno $(62,7 \%, 63,6 \%$, $77,0 \%$ y 76,7\%), mientras que para el 2018 Ucayali ocupó la última posición con un $68,1 \%$.

La variabilidad entre el 2013 y el 2018 identifica que la región con mayor incremento porcentual de AFC fue Cusco $(35,6 \%)$, otras dieciséis regiones también mejoraron a diferentes magnitudes, cuatro regiones (Ica, Huánuco, Junín, Cajamarca) no tuvieron variaciones importantes, mientras que otras cuatro regiones presentaron una reducción entre el -1,0\% al -12,5\% (Figura 1).

Los cambios porcentuales se relacionaron con el año evaluado, se encontró que la región Ayacucho mostró el coeficiente de correlación más elevado ( $r=0,912$ y $p=0,011$ ), esto expresa una variación acompañada de forma creciente con los años, la región con menor coeficiente de correlación fue Tacna ( $r=-0,018 ; p=0,011)$; los datos muestran que los cambios se presentan de forma diferenciada para cada ámbito geográfico y no siempre han ido acorde al paso de los años.

El análisis de tendencia (Figura 2) permitió identificar un valor APC de 1,06\% (IC 95\%: 0,49\%; 1,63\%); dicho análisis permite demostrar que hay una tendencia lineal monótona (de comportamiento único) de forma ascendente en los datos recolectados de forma transversal repetida a lo largo de los años $(p<0,001)$.

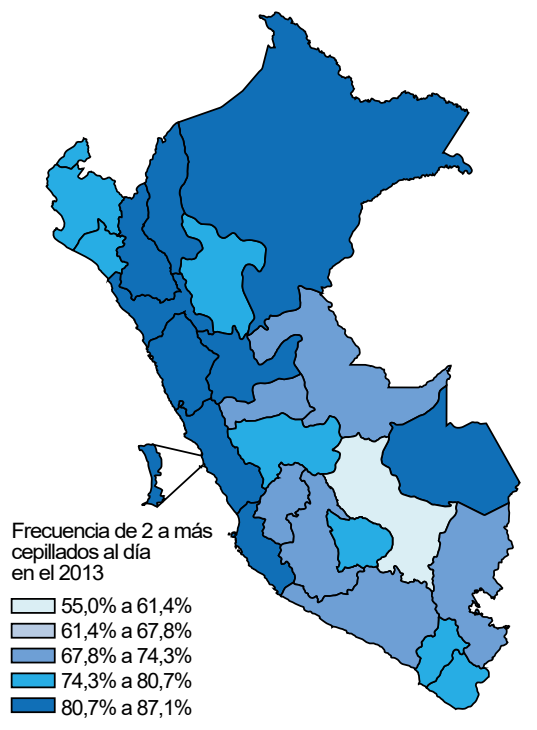

Con respecto a la distribución de AFC según sus características (Tabla 2), se encontró que todos los aspectos sanitarios considerados de forma bivariada se presentaban positivamente asociados, aunque sólo el haber recibido información sobre higiene bucal $(r=0,924$; $p=0,009)$ y el contar con seguro por EsSalud $(r=0,880$; $p=0,021)$ fueron factores que mostraron permanente asociación significativa en todos los años $(p<0,05)$. Adicionalmente, se evidencia que el contar con seguro militar de salud ha modificado en $22,3 \%$ la AFC entre el 2013 y $2018(p=0,039)$.

En consideración a las características de vivienda, sobre el tipo de lugar de residencia, el vivir en área urbana fue favorable para la AFC con un comportamiento creciente a lo largo de los años ( $r=0,938 ; p=0,006)$. Desde el 2014 al 2018 se ha encontrado que el quintil superior de bienestar socioeconómico se ha destacado en AFC con respecto a los demás; se identificó la tendencia que mientras menor el quintil, es menor el porcentaje de AFC $(p<0,001)$. En la evaluación de las características del hogar el único factor que presentó asociación favorable en la mayor cantidad de años (cuatro de cinco) fue el grupo etario en el que se encontraba el niño entre 2014 al 2018 ( $p<0,001)$, lo que se ha intensificado con el paso del tiempo en mayor medida para quienes se encuentran entre los tres y cinco años $(r=0,955 ; p=0,003)$.

En el modelo logístico multivariado (Tabla 3) se encontró que el paso del tiempo incrementó directamente, aunque no de forma armónica, la probabilidad de AFC respecto al 2013; todas las características sanitarias demostraron ser estadísticamente significativas, destacando el haber recibido

La diferencia relativa se calculó al ajustarla por el valor del 2013 (diferencia 2018-2013/valor del 2013 x 100).

Figura 1. Distribución por regiones para la adecuada frecuencia de cepillado dental en niños menores de 12 años y su diferencia relativa ENDES Perú 2013-2018. 


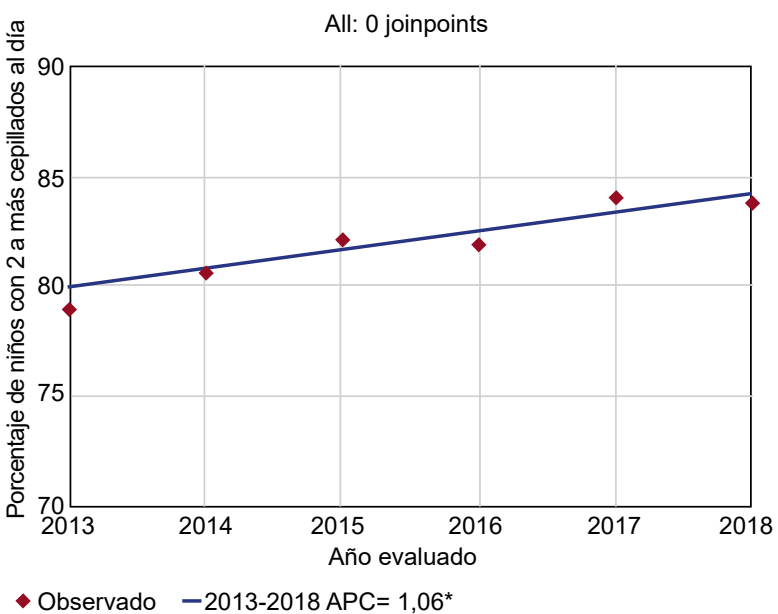

- Observado $-2013-2018$ APC $=1,06^{*}$

* Indica que el porcentaje anual de cambio (Anual Percent Change - APC) es significativamente diferente de cero con un nivel de significancia $=0,05$

Figura 2. Tendencia de la adecuada frecuencia de cepillado dental en niños menores de 12 años en Perú 2013-2018

atención odontológica previa (OR 1,37, IC 95\%: 1,30 a 1,44), así como información de higiene bucal (OR 1,38, IC 95\%: 1,31 a 1,45). El hecho de no compartir el cepillo es favorable para la AFC (OR 2,30, IC 95\%: 1,46 a 3,60). Las características de vivienda evidencian que, con respecto a la región natural, los niños de la sierra tienen menor probabilidad de AFC con respecto a los de Lima Metropolitana; geográficamente vivir en una zona rural repercute negativamente en la AFC (OR 0,87, IC 95\%: 0,81 a 0,94) con respecto a la urbana. Todos los quintiles de riqueza evaluados demostraron repercutir negativamente en la AFC con respecto a los niños que se encuentran en el quintil superior. El grupo etario fue el único factor del hogar que se vinculaba de forma significativa a la frecuencia de higiene presentándose de forma directamente proporcional, donde a mayor la edad del niño mayor la probabilidad de AFC tomando como categoría de referencia al grupo de uno a dos años.

\section{DISCUSIÓN}

La mejora en los hábitos de cepillado es de vital importancia para prevenir las enfermedades más comunes, estableciendo dos cepillados al día como un buen indicador de vida saludable ${ }^{(24)}$. Esta investigación reporta la creciente tendencia en la AFC en el Perú, así como en sus diferentes regiones, siendo las poblaciones de bajo estrato socioeconómico las que menor AFC presentaban.

El uso de servicios de salud en poblaciones rurales es menor a las de las poblaciones urbanas y son las primeras que muestran proporciones más bajas de AFC ${ }^{(25)}$. Las visitas al odontólogo se incrementan con la edad, el estrato socioeconómico y los ingresos mensuales de la familia, principalmente demandando evaluaciones, tratamientos restauradores $y / 0$ especializados que los obliga a ser referidos a centros hospitalarios.
Los países en vías de desarrollo, similares al Perú, muestran valores inferiores de AFC, un ejemplo es el reporte iraní en el que $75 \%$ de niños en edad preescolar cepillan sus dientes al menos una vez al día; en dicho estudio, el $28 \%$ de los casos el cepillado inició antes de los dos años de edad. Hay evidencia sobre la correlación entre el índice de higiene oral y la calidad de vida relacionada a la salud bucal ${ }^{(26)}$. Adicionalmente, una revisión sistemática establece que los niños con uno o más dientes con lesiones de caries genera consecuencias negativas en el desempeño y asistencia escolar cuando se comparan con niños con dentición saludable (27); tendencias de AFC en países desarrollados muestran mejor adherencia educativa ${ }^{(28)}$. En la mayoría de las investigaciones, al igual que en el presente estudio, las frecuencias de cepillado incrementan con la edad.

Estudios de tendencias muestran un alza en porcentajes de individuos con una AFC con el paso del tiempo, similares a las encontradas en el presente trabajo. En República Checa, un reporte de tendencias entre 1994 y 2014 sobre la frecuencia de cepillado dental en niños, mostró a través de una regresión logística binominal que esta incrementaba en todos los grupos evaluados, mostrando una mayor tasa en el grupo etario de doce años ${ }^{(28)}$, hallazgo independiente del estrato socio-económico, algo que no se cumplió en el presente estudio.

En países escandinavos, las proporciones de AFC son cercanas al $100 \%$. A pesar de la alta tasa de adherencia, las encuestas de tendencias realizadas en Noruega cada cuatro años (entre 1985 y 1997), mostraron que los niños entre 11 y 15 años tenían ligeros incrementos en la AFC con variabilidad estadísticamente significativa para los años 93 y 97 . Los encuestados mostraron valores de AFC por encima del $95 \%$ en todos los años incluidos en el reporte ${ }^{(29)}$. En Dinamarca, al igual que con el presente trabajo, las tasas de adherencia a una AFC era superior en los niños y adolescentes de estrato socio-económico alto ${ }^{(30)}$, incrementando la brecha de inequidades sociales para el mantenimiento de la salud.

La determinación del éxito de una intervención en salud pública requiere la obtención de información basal sobre el problema a abordar de manera que se pueda valorar el impacto del cambio generado durante y posterior de la intervención. Si bien, las encuestas nacionales representan herramientas que permiten cumplir con dicho objetivo, estas aún presentan sesgos inherentes a su metodología, ya sea durante el proceso de recolección de datos o por la alta variabilidad de la muestra. Es importante considerar que los datos reportados por la ENDES son de tipo muestral, por lo que estos pueden proporcionar una imagen optimista de la verdadera problemática alrededor de la AFC; la variabilidad propia de la higiene bucal en los niños dentro y entre 
Tabla 2. Distribución de la adecuada frecuencia de cepillado dental en niños menores de 12 años según características sanitarias de la vivienda y del hogar. Perú 2013-2018

\begin{tabular}{|c|c|c|c|c|c|c|c|c|c|c|c|c|}
\hline \multirow[b]{2}{*}{ Características } & \multicolumn{2}{|c|}{2013} & \multicolumn{2}{|l|}{2014} & \multicolumn{2}{|c|}{2015} & \multicolumn{2}{|l|}{2016} & \multicolumn{2}{|c|}{2017} & \multicolumn{2}{|l|}{2018} \\
\hline & $\begin{array}{c}\% \\
\text { (IC 95\%) }\end{array}$ & $\begin{array}{c}\text { Valor } \\
\mathbf{p}^{*}\end{array}$ & $\begin{array}{c}\% \\
\text { (IC 95\%) }\end{array}$ & $\begin{array}{l}\text { Valor } \\
\mathbf{p}^{*}\end{array}$ & $\begin{array}{c}\% \\
\text { (IC 95\%) }\end{array}$ & $\begin{array}{c}\text { Valor } \\
\mathbf{p}^{*}\end{array}$ & $\begin{array}{c}\% \\
\text { (IC 95\%) }\end{array}$ & $\begin{array}{c}\text { Valor } \\
\mathbf{p}^{*}\end{array}$ & $\begin{array}{c}\% \\
\text { (IC 95\%) }\end{array}$ & $\begin{array}{c}\text { Valor } \\
\mathbf{p}^{*}\end{array}$ & $\begin{array}{c}\% \\
\text { (IC 95\%) } \\
\end{array}$ & $\begin{array}{c}\text { Valor } \\
\mathbf{p}^{*}\end{array}$ \\
\hline \multicolumn{13}{|l|}{$\begin{array}{l}\text { Alguna vez atendido en el } \\
\text { servicio de odontología }\end{array}$} \\
\hline No & $\begin{array}{c}76,8 \\
(74,0 \text { a } 79,4)\end{array}$ & 0,059 & $\begin{array}{c}75,9 \\
(74,3 \text { a } 77,5)\end{array}$ & $<0,001$ & $\begin{array}{c}78,6 \\
(77,4 \text { a } 79,8)\end{array}$ & $<0,001$ & $\begin{array}{c}77,0 \\
(75,4 \text { a } 78,5)\end{array}$ & $<0,001$ & $\begin{array}{c}79,6 \\
(78,3 \mathrm{a} 80,8)\end{array}$ & $<0,001$ & $\begin{array}{c}79,6 \\
(78,5 \mathrm{a} 80,7)\end{array}$ & $<0,001$ \\
\hline $\mathrm{Si}$ & $\begin{array}{c}80,8 \\
(77,4 \text { a } 83,9)\end{array}$ & & $\begin{array}{c}84,2 \\
(83,0 \text { a } 85,2)\end{array}$ & & $\begin{array}{c}84,8 \\
(83,9 \text { a } 85,7)\end{array}$ & & $\begin{array}{c}85,8 \\
(85,0 \mathrm{a} 86,7)\end{array}$ & & $\begin{array}{c}86,3 \\
(85,5 \text { a } 87,1)\end{array}$ & & $\begin{array}{c}86,1 \\
(85,3 \mathrm{a} 86,9)\end{array}$ & \\
\hline No & $\begin{array}{c}76,7 \\
(73,3 \text { a } 79,8)\end{array}$ & 0,005 & $\begin{array}{c}78,2 \\
(76,6 \text { a } 79,8)\end{array}$ & $<0,001$ & $\begin{array}{c}79,3 \\
(77,9 a \mathrm{a} 80,7)\end{array}$ & $<0,001$ & $\begin{array}{c}79,6 \\
(78,2 \mathrm{a} 81,0)\end{array}$ & $<0,001$ & $\begin{array}{c}81,8 \\
(80,4 \text { a } 83,0)\end{array}$ & $<0,001$ & $\begin{array}{c}81,3 \\
(80,1 \mathrm{a} 82,4)\end{array}$ & $<0,001$ \\
\hline $\mathrm{Si}$ & $\begin{array}{c}82,4 \\
(79,6 \text { a } 84,9)\end{array}$ & & $\begin{array}{c}84,0 \\
(82,8 \mathrm{a} 85,2)\end{array}$ & & $\begin{array}{c}85,6 \\
(84,7 \text { a } 86,4)\end{array}$ & & $\begin{array}{c}85,5 \\
(84,6 \text { a } 86,4)\end{array}$ & & $\begin{array}{c}86,6 \\
(85,7 a \mathrm{a} 87,5)\end{array}$ & & $\begin{array}{c}86,4 \\
(85,6 \text { a } 87,2)\end{array}$ & \\
\hline No sabe / No Recuerda & $\begin{array}{c}74,0 \\
(49,5 \text { a } 89,2)\end{array}$ & & $\begin{array}{c}78,4 \\
(65,8 \text { a } 87,2)\end{array}$ & & $\begin{array}{c}77,3 \\
(64,9 a \mathrm{a} 86,2)\end{array}$ & & $\begin{array}{c}81,2 \\
(65,7 \text { a } 90,6)\end{array}$ & & $\begin{array}{c}83,5 \\
(63,7 \text { a } 93,5)\end{array}$ & & $\begin{array}{c}79,9 \\
(61,5 \text { a } 90,9)\end{array}$ & \\
\hline Responde el mismo niño & $\begin{array}{c}76,5 \\
(72,4 \text { a } 80,2)\end{array}$ & & $\begin{array}{c}78,0 \\
(76,3 \text { a } 79,7)\end{array}$ & & $\begin{array}{c}80,0 \\
(78,7 \text { a } 81,2)\end{array}$ & & $\begin{array}{c}79,6 \\
(78,2 \mathrm{a} 81,0)\end{array}$ & & $\begin{array}{c}81,9 \\
(80,6 ; a 83,2)\end{array}$ & & $\begin{array}{c}82,3 \\
(81,1 \text { a } 83,4)\end{array}$ & \\
\hline $\mathrm{Si}$ & $\begin{array}{c}51,0 \\
(22,8 \mathrm{a} 78,6)\end{array}$ & 0,032 & $\begin{array}{c}61,9 \\
(42,9 \text { a } 77,9)\end{array}$ & 0,014 & $\begin{array}{c}54,7 \\
(29,7 \text { a } 77,5)\end{array}$ & 0,007 & $\begin{array}{c}72,9 \\
(56,9 \text { a } 84,5)\end{array}$ & 0,136 & $\begin{array}{c}79,7 \\
(59,6 \text { a } 91,2)\end{array}$ & 0,555 & $\begin{array}{c}54,5 \\
(27,7 ; 78,9)\end{array}$ & 0,006 \\
\hline No & $\begin{array}{c}79,1 \\
(76,8 \mathrm{a} 81,3)\end{array}$ & & $\begin{array}{c}80,7 \\
(79,7 \text { a } 81,8)\end{array}$ & & $\begin{array}{c}82,2 \\
(81,4 \text { a } 83,0)\end{array}$ & & $\begin{array}{c}82,0 \\
(81,1 \mathrm{a} 82,9)\end{array}$ & & $\begin{array}{c}84,0 \\
(83,3 \mathrm{a} 84,7)\end{array}$ & & $\begin{array}{c}83,9 \\
(83,2 ; 84,6)\end{array}$ & \\
\hline \multicolumn{13}{|l|}{ Usa dentífrico para cepillarse } \\
\hline No & $\begin{array}{c}66,0 \\
(47,9 \mathrm{a} 80,4)\end{array}$ & 0,075 & $\begin{array}{c}61,5 \\
(53,0 \text { a } 69,3)\end{array}$ & $<0,001$ & $\begin{array}{c}64,8 \\
(58,3 \text { a } 70,7)\end{array}$ & $<0,001$ & $\begin{array}{c}65,8 \\
(59,0 \text { a } 72,0)\end{array}$ & $<0,001$ & $\begin{array}{c}65,0 \\
(57,9 \text { a } 71,5)\end{array}$ & $<0,001$ & $\begin{array}{c}70,8 \\
(64,9 \text { a } 76,0)\end{array}$ & $<0,001$ \\
\hline $\mathrm{Si}$ & $\begin{array}{c}79,2 \\
(76,9 \text { a } 81,4)\end{array}$ & & $\begin{array}{c}80,9 \\
(79,8 \text { a } 81,9)\end{array}$ & & $\begin{array}{c}82,2 \\
(81,4 \mathrm{a} 83,0)\end{array}$ & & $\begin{array}{c}82,1 \\
(81,2 \mathrm{a} 83,0)\end{array}$ & & $\begin{array}{c}84,2 \\
(83,5 \mathrm{a} 85,0)\end{array}$ & & $\begin{array}{c}84,0 \\
(83,4 \text { a } 84,7)\end{array}$ & \\
\hline \multicolumn{13}{|l|}{ Tiene algún tipo de seguro } \\
\hline No & $\begin{array}{c}77,6 \\
(73,1 \mathrm{a} 81,6)\end{array}$ & 0,388 & $\begin{array}{c}79,8 \\
(77,8 \text { a } 81,7)\end{array}$ & 0,264 & $\begin{array}{c}81,7 \\
(80,2 \mathrm{a} 83,2)\end{array}$ & 0,574 & $\begin{array}{c}81,4 \\
(79,5 \text { a } 83,2)\end{array}$ & 0,462 & $\begin{array}{c}82,1 \\
(80,5 \mathrm{a} 83,5)\end{array}$ & 0,002 & $\begin{array}{c}82,4 \\
(81,1 \mathrm{a} 83,7)\end{array}$ & 0,007 \\
\hline $\mathrm{Si}$ & $\begin{array}{c}79,7 \\
(77,1 \mathrm{a} 82,0)\end{array}$ & & $\begin{array}{c}81,0 \\
(79,8 \text { a } 82,1)\end{array}$ & & $\begin{array}{c}82,2 \\
(81,3 \text { a } 83,1)\end{array}$ & & $\begin{array}{c}82,2 \\
(81,2 \mathrm{a} 83,1)\end{array}$ & & $\begin{array}{c}84,7 \\
(83,8 \text { a } 85,5)\end{array}$ & & $\begin{array}{c}84,3 \\
(83,6 \text { a } 85,1)\end{array}$ & \\
\hline \multicolumn{13}{|l|}{$\begin{array}{l}\text { Cuenta con seguro por } \\
\text { Essalud }\end{array}$} \\
\hline No & $\begin{array}{c}77,7 \\
(75,2 \mathrm{a} 80,0)\end{array}$ & 0,020 & $\begin{array}{c}79,3 \\
(78,1 \text { a } 80,4)\end{array}$ & $<0,001$ & $\begin{array}{c}81,3 \\
(80,3 \mathrm{a} 82,2)\end{array}$ & $<0,001$ & $\begin{array}{c}81,4 \\
(80,3 \mathrm{a} 82,4)\end{array}$ & 0,001 & $\begin{array}{c}83,2 \\
(82,3 \mathrm{a} 84,0)\end{array}$ & $<0,001$ & $\begin{array}{c}82,7 \\
(81,9 \mathrm{a} 83,5)\end{array}$ & $<0,001$ \\
\hline No & $\begin{array}{c}79,2 \\
(76,9 \mathrm{a} 81,3)\end{array}$ & 0,086 & $\begin{array}{c}80,6 \\
(79,5 \text { a } 81,6)\end{array}$ & 0,068 & $\begin{array}{c}82,0 \\
(81,2 \mathrm{a} 82,8)\end{array}$ & 0,008 & $\begin{array}{c}82,0 \\
(81,1 \mathrm{a} 82,8)\end{array}$ & 0,159 & $\begin{array}{c}83,9 \\
(83,2 \mathrm{a} 84,7)\end{array}$ & 0,045 & $\begin{array}{c}83,8 \\
(83,2 \mathrm{a} 84,5)\end{array}$ & 0,459 \\
\hline $\mathrm{Si}$ & $\begin{array}{c}64,1 \\
(42,6 \text { a } 81,1)\end{array}$ & & $\begin{array}{c}87,4 \\
(80,0 \text { a } 92,4)\end{array}$ & & $\begin{array}{c}88,6 \\
(84,0 \text { a } 92,1)\end{array}$ & & $\begin{array}{c}85,9 \\
(80,3 \text { a } 90,1)\end{array}$ & & $\begin{array}{c}88,9 \\
(84,0 \text { a } 92,4)\end{array}$ & & $\begin{array}{c}86,4 \\
(78,8 \text { a } 91,6)\end{array}$ & \\
\hline \multicolumn{13}{|l|}{$\begin{array}{l}\text { Cuenta con Seguro Integral } \\
\text { de Salud }\end{array}$} \\
\hline No & $\begin{array}{c}79,9 \\
(76,5 \mathrm{a} 83,0)\end{array}$ & 0,340 & $\begin{array}{c}82,4 \\
(81,0 \text { a } 83,7)\end{array}$ & $<0,001$ & $\begin{array}{c}83,4 \\
(82,4 \text { a } 84,3)\end{array}$ & $<0,001$ & $\begin{array}{c}83,0 \\
(81,7 \text { a } 84,1)\end{array}$ & 0,043 & $\begin{array}{c}84,4 \\
(83,4 \text { a } 85,3)\end{array}$ & 0,222 & $\begin{array}{c}84,9 \\
(84,0 \text { a } 85,7)\end{array}$ & $<0,001$ \\
\hline $\mathrm{Si}$ & $\begin{array}{c}77,9 \\
(75,0 \text { a 80,5) }\end{array}$ & & $\begin{array}{c}78,5 \\
(77,0 \text { a } 79,9)\end{array}$ & & $\begin{array}{c}80,9 \\
(79,7 \text { a } 82,1)\end{array}$ & & $\begin{array}{c}81,2 \\
(79,9 a 82,4)\end{array}$ & & $\begin{array}{c}83,5 \\
(82,5 \text { a 84,5) }\end{array}$ & & $\begin{array}{c}82,5 \\
(81,5 \text { a 83,5) }\end{array}$ & \\
\hline \multicolumn{13}{|l|}{$\begin{array}{l}\text { Cuenta con seguro por } \\
\text { compañía aseguradora }\end{array}$} \\
\hline No & $\begin{array}{c}79,0 \\
(76,7 \text { a } 81,2)\end{array}$ & 0,809 & $\begin{array}{c}80,5 \\
(79,5 \text { a } 81,5)\end{array}$ & 0,003 & $\begin{array}{c}82,0 \\
(81,2 \text { a } 82,8)\end{array}$ & 0,019 & $\begin{array}{c}82,0 \\
(81,1 \text { a } 82,9)\end{array}$ & 0,464 & $\begin{array}{c}83,8 \\
(83,1 \mathrm{a} 84,5)\end{array}$ & $<0,001$ & $\begin{array}{c}83,8 \\
(83,1 \mathrm{a} 84,4)\end{array}$ & 0,131 \\
\hline $\mathrm{Si}$ & $\begin{array}{c}82,1 \\
(48,7 \text { a } 95,7)\end{array}$ & & $\begin{array}{c}93,5 \\
(85,6 \text { a } 97,2)\end{array}$ & & $\begin{array}{c}89,7 \\
(83,5 a 93,7)\end{array}$ & & $\begin{array}{c}79,4 \\
(70,9 \text { a 85,9) }\end{array}$ & & $\begin{array}{c}92,0 \\
(87,7 \text { a } 94,9)\end{array}$ & & $\begin{array}{c}88,7 \\
(82,0 \text { a } 93,1)\end{array}$ & \\
\hline \multicolumn{13}{|l|}{ Cuenta con seguro privado } \\
\hline No & $\begin{array}{c}78,9 \\
(76,6 \text { a 81,1) }\end{array}$ & 0,033 & $\begin{array}{c}80,6 \\
(79,5 \text { a } 81,6)\end{array}$ & 0,038 & $\begin{array}{c}82,1 \\
(81,2 \mathrm{a} 82,9)\end{array}$ & 0,475 & $\begin{array}{c}82,0 \\
(81,1 \mathrm{a} 82,9)\end{array}$ & 0,421 & $\begin{array}{c}84,0 \\
(83,2 \mathrm{a} 84,7)\end{array}$ & 0,172 & $\begin{array}{c}83,8 \\
(83,1 \mathrm{a} 84,5)\end{array}$ & 0,078 \\
\hline $\mathrm{Si}$ & $\begin{array}{c}96,4 \\
(76,5 \text { a } 99,5)\end{array}$ & & $\begin{array}{c}88,5 \\
(81,0 \text { a } 93,3)\end{array}$ & & $\begin{array}{c}84,6 \\
(76,9 \text { a } 90,1)\end{array}$ & & $\begin{array}{c}85,4 \\
(76,0 \text { a } 91,6)\end{array}$ & & $\begin{array}{c}88,4 \\
(81,6 \text { a } 92,9)\end{array}$ & & $\begin{array}{c}89,0 \\
(83,1 \mathrm{a} 93,0)\end{array}$ & \\
\hline \multicolumn{13}{|l|}{ Región natural } \\
\hline Lima metropolitana & $\begin{array}{c}78,7 \\
(71,5 \mathrm{a} 84,5)\end{array}$ & 0,151 & $\begin{array}{c}85,1 \\
(82,8 \text { a } 87,2)\end{array}$ & $<0,001$ & $\begin{array}{c}85,0 \\
(83,3 a \text { 86,5) }\end{array}$ & $<0,001$ & $\begin{array}{c}86,1 \\
(84,4 \text { a } 87,7)\end{array}$ & $<0,001$ & $\begin{array}{c}86,5 \\
(84,9 \text { a } 88,0)\end{array}$ & $<0,001$ & $\begin{array}{c}86,2 \\
(84,8 \text { a } 87,6)\end{array}$ & $<0,001$ \\
\hline Resto de Costa & $\begin{array}{c}82,7 \\
(78,7 \text { a } 86,0)\end{array}$ & & $\begin{array}{c}82,8 \\
(80,8 \text { a } 84,7)\end{array}$ & & $\begin{array}{c}84,0 \\
(82,7 \text { a } 85,2)\end{array}$ & & $\begin{array}{c}83,4 \\
(81,9 \text { a } 84,8)\end{array}$ & & $\begin{array}{c}85,5 \\
(84,3 \text { a } 86,6)\end{array}$ & & $\begin{array}{c}85,2 \\
(84,0 \text { a } 86,3)\end{array}$ & \\
\hline Sierra & $\begin{array}{c}75,7 \\
(72,1 \text { a } 79,0)\end{array}$ & & $\begin{array}{c}74,7 \\
(72,8 \text { a } 76,6)\end{array}$ & & $\begin{array}{c}78,5 \\
(77,0 \text { a } 79,9)\end{array}$ & & $\begin{array}{c}78,6 \\
(76,7 \text { a } 80,3)\end{array}$ & & $\begin{array}{c}79,5 \\
(78,0 \text { a } 80,9)\end{array}$ & & $\begin{array}{c}79,7 \\
(78,4 \text { a } 81,0)\end{array}$ & \\
\hline Selva & $\begin{array}{c}79,6 \\
(76,5 \text { a 82,4) } \\
\end{array}$ & & $\begin{array}{c}80,2 \\
(78,3 \text { a } 82,0)\end{array}$ & & $\begin{array}{c}81,9 \\
(79,9 a \mathrm{a} 83,7) \\
\end{array}$ & & $\begin{array}{c}81,5 \\
(79,5 a 83,3) \\
\end{array}$ & & $\begin{array}{c}83,5 \\
(82,0 \text { a 84,9) } \\
\end{array}$ & & $\begin{array}{c}83,3 \\
(82,1 \mathrm{a} 84,4) \\
\end{array}$ & \\
\hline
\end{tabular}

* Comparación con la proporción de cepillado 1 vez al día, mediante prueba Chi Cuadrado de Pearson.

** Diferencia establecida entre los estimadores porcentuales del año 2013 y 2018 mediante regresión logística binaria con link identity.

*** Correlación establecida entre los años y el estimador porcentual de 2 a más cepillados por día para cada categoría de la característica evaluada.

† Coeficiente de correlación de Pearson.

‡ Coeficiente de correlación de Spearman-Brown.

(Continuá en la página 569) 
Tabla 2. Distribución de la adecuada frecuencia de cepillado dental en niños menores de 12 años según características sanitarias de la vivienda y del hogar. Perú 2013-2018 (Viene de la página 568)

\begin{tabular}{|c|c|c|c|c|c|c|c|c|c|c|c|c|}
\hline \multirow[b]{2}{*}{ Características } & \multicolumn{2}{|l|}{2013} & \multicolumn{2}{|l|}{2014} & \multicolumn{2}{|l|}{2015} & \multicolumn{2}{|l|}{2016} & \multicolumn{2}{|c|}{2017} & \multicolumn{2}{|l|}{2018} \\
\hline & $\begin{array}{c}\% \\
\text { (IC 95\%) }\end{array}$ & $\underset{p^{*}}{\text { Valor }}$ & $\begin{array}{c}\% \\
\text { (IC 95\%) }\end{array}$ & $\begin{array}{c}\text { Valor } \\
\mathbf{p}^{*}\end{array}$ & $\begin{array}{c}\% \\
\text { (IC 95\%) }\end{array}$ & $\begin{array}{c}\text { Valor } \\
\mathbf{p}^{*}\end{array}$ & $\begin{array}{c}\% \\
\text { (IC } 95 \%)\end{array}$ & $\begin{array}{c}\text { Valor } \\
\mathbf{p}^{*}\end{array}$ & $\begin{array}{c}\% \\
\text { (IC 95\%) }\end{array}$ & $\begin{array}{c}\text { Valor } \\
\mathbf{p}^{*}\end{array}$ & $\begin{array}{c}\% \\
\text { (IC 95\%) }\end{array}$ & $\begin{array}{c}\text { Valor } \\
\mathbf{p}^{*}\end{array}$ \\
\hline \multicolumn{13}{|l|}{ Tipo de lugar de residencia } \\
\hline Urbano & $\begin{array}{c}81,0 \\
(77,9 \text { a } 83,7)\end{array}$ & 0,004 & $\begin{array}{c}82,4 \\
(81,1 ; 83,6)\end{array}$ & $<0,001$ & $\begin{array}{c}83,5 \\
(82,7 \text { a } 84,3)\end{array}$ & $<0,001$ & $\begin{array}{c}83,6 \\
(82,7 \text { a } 84,4)\end{array}$ & $<0,001$ & $\begin{array}{c}84,9 \\
(84,1 \text { a } 85,7)\end{array}$ & $<0,001$ & $\begin{array}{c}84,5 \\
(83,7 \text { a } 85,3)\end{array}$ & $<0,001$ \\
\hline Rural & $\begin{array}{c}74,7 \\
(71,5 \mathrm{a} 77,7)\end{array}$ & & $\begin{array}{c}76,3 \\
(74,6 ; 78,0)\end{array}$ & & $\begin{array}{c}80,1 \\
(78,5 \text { a } 81,5)\end{array}$ & & $\begin{array}{c}79,9 \\
(78,1 \text { a } 81,5)\end{array}$ & & $\begin{array}{c}81,2 \\
(79,6 \text { a } 82,6)\end{array}$ & & $\begin{array}{c}81,7 \\
(80,4 \text { a } 83,0)\end{array}$ & \\
\hline \multicolumn{13}{|l|}{ Quintil de riqueza } \\
\hline Quintil inferior & $\begin{array}{c}74,0 \\
(70,1 \mathrm{a} 77,5)\end{array}$ & 0,107 & $\begin{array}{c}77,4 \\
(75,5 ; 79,3)\end{array}$ & $<0,001$ & $\begin{array}{c}80,8 \\
(79,1 \text { a } 82,4)\end{array}$ & $<0,001$ & $\begin{array}{c}80,8 \\
(78,9 a 82,5)\end{array}$ & $<0,001$ & $\begin{array}{c}82,1 \\
(80,5 \mathrm{a} 83,5)\end{array}$ & $<0,001$ & $\begin{array}{c}83,0 \\
(81,7 \text { a } 84,2)\end{array}$ & $<0,001$ \\
\hline Segundo quintil & $\begin{array}{c}79,5 \\
(75,4 \text { a } 83,1)\end{array}$ & & $\begin{array}{c}77,6 \\
(75,6 ; 79,5)\end{array}$ & & $\begin{array}{c}80,4 \\
(78,9 \text { a } 81,8)\end{array}$ & & $\begin{array}{c}80,2 \\
(78,6 \text { a } 81,7)\end{array}$ & & $\begin{array}{c}83,3 \\
(81,8 \mathrm{a} 84,6)\end{array}$ & & $\begin{array}{c}81,6 \\
(80,1 \mathrm{a} 83,1)\end{array}$ & \\
\hline Quintil intermedio & $\begin{array}{c}80,6 \\
(75,2 \mathrm{a} 85,1)\end{array}$ & & $\begin{array}{c}80,3 \\
(78,0 ; 82,4)\end{array}$ & & $\begin{array}{c}82,7 \\
(81,2 \text { a } 84,2)\end{array}$ & & $\begin{array}{c}81,6 \\
(80,1 \text { a } 83,1)\end{array}$ & & $\begin{array}{c}83,2 \\
(81,5 \text { a } 84,7)\end{array}$ & & $\begin{array}{c}82,9 \\
(81,4 \text { a } 84,4)\end{array}$ & \\
\hline Cuarto quintil & $\begin{array}{c}83,2 \\
(76,8 \mathrm{a} 88,1)\end{array}$ & & $\begin{array}{c}82,2 \\
(79,9 ; 84,3)\end{array}$ & & $\begin{array}{c}83,5 \\
(81,8 \text { a } 85,1)\end{array}$ & & $\begin{array}{c}85,1 \\
(83,4 \text { a } 86,6)\end{array}$ & & $\begin{array}{c}84,9 \\
(83,0 \text { a } 86,7)\end{array}$ & & $\begin{array}{c}85,4 \\
(83,8 \mathrm{a} 86,9)\end{array}$ & \\
\hline Quintil superior & $\begin{array}{c}78,4 \\
(71,0 \text { a } 84,3)\end{array}$ & & $\begin{array}{c}88,3 \\
(86,2 ; 90,2)\end{array}$ & & $\begin{array}{c}87,3 \\
(85,7 \text { a } 88,8)\end{array}$ & & $\begin{array}{c}87,5 \\
(85,6 \text { a } 89,1)\end{array}$ & & $\begin{array}{c}88,1 \\
(86,2 \mathrm{a} 89,7)\end{array}$ & & $\begin{array}{c}87,9 \\
(86,2 \text { a } 89,4)\end{array}$ & \\
\hline \multicolumn{13}{|l|}{ Hogar con bomba de agua } \\
\hline No tiene & $\begin{array}{c}78,8 \\
(76,3 \mathrm{a} 81,0)\end{array}$ & 0,203 & $\begin{array}{c}80,5 \\
(79,4 ; 81,5)\end{array}$ & 0,103 & $\begin{array}{c}82,0 \\
(81,2 \text { a } 82,8)\end{array}$ & 0,400 & $\begin{array}{c}81,8 \\
(80,8 \mathrm{a} 82,7)\end{array}$ & $<0,001$ & $\begin{array}{c}84,1 \\
(83,3 \text { a } 84,8)\end{array}$ & 0,633 & $\begin{array}{c}83,6 \\
(82,9 \text { a } 84,3)\end{array}$ & 0,002 \\
\hline Si tiene & $\begin{array}{c}86,3 \\
(73,9 \text { a } 93,3)\end{array}$ & & $\begin{array}{c}83,7 \\
(79,9 \text { a } 87,0)\end{array}$ & & $\begin{array}{c}83,8 \\
(79,4 \text { a } 87,5)\end{array}$ & & $\begin{array}{c}87,3 \\
(84,7 \text { a } 89,5)\end{array}$ & & $\begin{array}{c}83,3 \\
(79,7 \text { a } 86,3)\end{array}$ & & $\begin{array}{c}87,4 \\
(85,2 \mathrm{a} 89,4)\end{array}$ & \\
\hline \multicolumn{13}{|l|}{ Estructura del hogar } \\
\hline Sin adultos & $\begin{array}{c}48,9 \\
(20,3 \text { a } 78,2)\end{array}$ & 0,205 & $\begin{array}{c}100,0 \\
(100,0 \text { a 100,0) }\end{array}$ & 0,294 & $\begin{array}{c}99,8 \\
(98,1 \mathrm{a} 100,0)\end{array}$ & 0,293 & - & 0,764 & - & 0,381 & - & 0,118 \\
\hline Un adulto & $\begin{array}{c}79,2 \\
(71,8 \mathrm{a} 85,0)\end{array}$ & & $\begin{array}{c}78,9 \\
(75,1 ; 82,2)\end{array}$ & & $\begin{array}{c}84,5 \\
(81,5 \text { a } 87,1)\end{array}$ & & $\begin{array}{c}81,1 \\
(77,7 \mathrm{a} 84,1)\end{array}$ & & $\begin{array}{c}83,4 \\
(80,8 \text { a } 85,7)\end{array}$ & & $\begin{array}{c}82,3 \\
(79,4 \text { a } 84,9)\end{array}$ & \\
\hline $\begin{array}{l}\text { Dos adultos de sexo } \\
\text { opuesto }\end{array}$ & $\begin{array}{c}78,8 \\
(75,6 \text { a } 81,8)\end{array}$ & & $\begin{array}{c}81,0 \\
(79,6 \text { a } 82,4)\end{array}$ & & $\begin{array}{c}82,0 \\
(80,9 \text { a } 83,1)\end{array}$ & & $\begin{array}{c}82,1 \\
(80,8 \text { a } 83,3)\end{array}$ & & $\begin{array}{c}84,3 \\
(83,2 \mathrm{a} 85,3)\end{array}$ & & $\begin{array}{c}84,7 \\
(83,8 \text { a } 85,6)\end{array}$ & \\
\hline $\begin{array}{l}\text { Dos adultos del mismo } \\
\text { sexo }\end{array}$ & $\begin{array}{c}88,6 \\
(79,6 \text { a } 94,0)\end{array}$ & & $\begin{array}{c}76,7 \\
(70,1 \text { a } 82,2)\end{array}$ & & $\begin{array}{c}79,5 \\
(74,8 \text { a } 83,6)\end{array}$ & & $\begin{array}{c}81,4 \\
(76,5 \mathrm{a} 85,5)\end{array}$ & & $\begin{array}{c}81,7 \\
(76,0 \text { a } 86,3)\end{array}$ & & $\begin{array}{c}80,4 \\
(75,6 \text { a } 84,5)\end{array}$ & \\
\hline $\begin{array}{l}\text { Tres o más adultos } \\
\text { relacionados }\end{array}$ & $\begin{array}{c}78,3 \\
(74,6 \text { a } 81,5)\end{array}$ & & $\begin{array}{c}80,5 \\
(79,1 \mathrm{a} 81,9)\end{array}$ & & $\begin{array}{c}82,3 \\
(81,1 \text { a } 83,4)\end{array}$ & & $\begin{array}{c}82,2 \\
(80,9 \text { a } 83,4)\end{array}$ & & $\begin{array}{c}83,7 \\
(82,6 \text { a } 84,8)\end{array}$ & & $\begin{array}{c}83,4 \\
(82,4 \text { a } 84,5)\end{array}$ & \\
\hline Adultos no relacionados & $\begin{array}{c}86,2 \\
(73,5 \text { a } 93,4)\end{array}$ & & $\begin{array}{c}84,2 \\
(78,8 \text { a } 88,4)\end{array}$ & & $\begin{array}{c}77,9 \\
(68,4 \text { a } 85,2)\end{array}$ & & $\begin{array}{c}79,2 \\
(73,1 \mathrm{a} 84,3)\end{array}$ & & $\begin{array}{c}87,4 \\
(83,0 \text { a } 90,8)\end{array}$ & & $\begin{array}{c}82,9 \\
(77,7 \text { a } 87,1)\end{array}$ & \\
\hline \multicolumn{13}{|l|}{ Sexo de la cabeza de familia } \\
\hline Masculino & $\begin{array}{c}77,9 \\
(75,2 \mathrm{a} 80,4)\end{array}$ & 0,024 & $\begin{array}{c}81,0 \\
(79,9 \text { a } 82,0)\end{array}$ & 0,163 & $\begin{array}{c}81,9 \\
(80,9 \text { a } 82,8)\end{array}$ & 0,152 & $\begin{array}{c}82,1 \\
(81,1 \mathrm{a} 83,1)\end{array}$ & 0,390 & $\begin{array}{c}84,2 \\
(83,0 \text { a } 4,85)\end{array}$ & 0,345 & $\begin{array}{c}84,0 \\
(83,2 \text { a } 84,8)\end{array}$ & 0,457 \\
\hline Femenino & $\begin{array}{c}83,4 \\
(79,2 \mathrm{a} 86,9)\end{array}$ & & $\begin{array}{c}79,4 \\
(77,1 \text { a } 81,5)\end{array}$ & & $\begin{array}{c}83,1 \\
(81,6 \text { a } 84,6)\end{array}$ & & $\begin{array}{c}81,3 \\
(79,5 \text { a } 83,0)\end{array}$ & & $\begin{array}{c}83,4 \\
(81,8 \text { a } 84,9)\end{array}$ & & $\begin{array}{c}83,4 \\
(81,9 \text { a } 84,8)\end{array}$ & \\
\hline \multicolumn{13}{|l|}{$\begin{array}{l}\text { Nivel educativo de la cabeza } \\
\text { del hogar }\end{array}$} \\
\hline Sin educación & $\begin{array}{c}77,0 \\
(72,3 \mathrm{a} 81,0)\end{array}$ & 0,417 & $\begin{array}{c}78,6 \\
(76,4 \text { a } 80,5)\end{array}$ & $<0,001$ & $\begin{array}{c}81,1 \\
(79,7 \text { a } 82,4)\end{array}$ & 0,086 & $\begin{array}{c}81,6 \\
(79,9 \text { a } 83,2)\end{array}$ & 0,086 & $\begin{array}{c}83,7 \\
(82,4 \text { a } 84,9)\end{array}$ & 0,174 & $\begin{array}{c}84,1 \\
(82,9 \text { a } 85,2)\end{array}$ & 0,027 \\
\hline Primaria & $\begin{array}{c}80,8 \\
(77,3 \mathrm{a} 84,0)\end{array}$ & & $\begin{array}{c}79,8 \\
(78,1 \mathrm{a} 81,4)\end{array}$ & & $\begin{array}{c}81,9 \\
(80,4 \text { a } 83,2)\end{array}$ & & $\begin{array}{c}81,6 \\
(79,9 \text { a } 83,1)\end{array}$ & & $\begin{array}{c}85,0 \\
(83,8 \mathrm{a} 86,2)\end{array}$ & & $\begin{array}{c}83,3 \\
(81,9 \mathrm{a} 84,5)\end{array}$ & \\
\hline Secundaria & $\begin{array}{c}77,8 \\
(73,3 \text { a } 81,8)\end{array}$ & & $\begin{array}{c}81,6 \\
(79,9 \text { a } 83,1)\end{array}$ & & $\begin{array}{c}82,9 \\
(81,1 \text { a } 84,5)\end{array}$ & & $\begin{array}{c}81,9 \\
(80,5 \text { a } 83,2)\end{array}$ & & $\begin{array}{c}83,0 \\
(81,6 \text { a } 84,3)\end{array}$ & & $\begin{array}{c}83,0 \\
(81,6 \text { a } 84,2)\end{array}$ & \\
\hline Superior & $\begin{array}{c}81,0 \\
(75,2 \text { a } 85,7)\end{array}$ & & $\begin{array}{c}84,5 \\
(82,3 \text { a } 86,5)\end{array}$ & & $\begin{array}{c}83,5 \\
(81,7 \text { a } 85,2)\end{array}$ & & $\begin{array}{c}84,9 \\
(83,0 \text { a } 86,6)\end{array}$ & & $\begin{array}{c}84,3 \\
(82,3 \text { a } 86,1)\end{array}$ & & $\begin{array}{c}85,8 \\
(84,3 \text { a } 87,2)\end{array}$ & \\
\hline \multicolumn{13}{|c|}{$\begin{array}{l}\text { Cabeza del hogar estudiando } \\
\text { en el año de evaluación }\end{array}$} \\
\hline No estudia & $\begin{array}{c}78,6 \\
(75,5 \text { a 81,3) }\end{array}$ & 0,588 & $\begin{array}{c}81,0 \\
(79,7 \text { a } 82,1)\end{array}$ & 0,430 & $\begin{array}{c}81,7 \\
(80,6 \text { a } 82,8)\end{array}$ & 0,350 & $\begin{array}{c}81,9 \\
(80,9 \text { a } 83,0)\end{array}$ & 0,857 & $\begin{array}{c}83,5 \\
(82,6 \text { a } 84,4)\end{array}$ & 0,057 & $\begin{array}{c}83,6 \\
(82,8 \text { a } 84,4)\end{array}$ & 0,224 \\
\hline Si estudia & $\begin{array}{c}79,8 \\
(76,2 \mathrm{a} 82,9)\end{array}$ & & $\begin{array}{c}80,2 \\
(78,7 \text { a } 81,7)\end{array}$ & & $\begin{array}{c}82,6 \\
(81,2 \text { a 83,8) }\end{array}$ & & $\begin{array}{c}82,1 \\
(80,6 \text { a } 83,5)\end{array}$ & & $\begin{array}{c}84,9 \\
(83,7 \text { a } 86,0)\end{array}$ & & $\begin{array}{c}84,4 \\
(83,3 \text { a } 85,5)\end{array}$ & \\
\hline \multicolumn{13}{|l|}{ Grupo etario del niño (años) } \\
\hline 1 a 2 & $\begin{array}{c}74,1 \\
(67,3 \text { a 80,0) }\end{array}$ & 0,168 & $\begin{array}{c}70,7 \\
(67,5 \text { a } 73,8)\end{array}$ & $<0,001$ & $\begin{array}{c}72,5 \\
(70,2 \mathrm{a} 74,7)\end{array}$ & $<0,001$ & $\begin{array}{c}71,2 \\
(69,0 \text { a } 73,3)\end{array}$ & $<0,001$ & $\begin{array}{c}73,7 \\
(71,7 a 75,6)\end{array}$ & $<0,001$ & $\begin{array}{c}73,9 \\
(72,1 \mathrm{a} 75,6)\end{array}$ & $<0,001$ \\
\hline 3 a 5 & $\begin{array}{c}78,0 \\
(74,3 \mathrm{a} 81,3)\end{array}$ & & $\begin{array}{c}77,8 \\
(76,0 \text { a } 79,5)\end{array}$ & & $\begin{array}{c}80,5 \\
(79,4 ; 81,7)\end{array}$ & & $\begin{array}{c}80,4 \\
(79,1 \mathrm{a} 81,6)\end{array}$ & & $\begin{array}{c}83,2 \\
(82,1 \text { a } 84,3)\end{array}$ & & $\begin{array}{c}83,6 \\
(82,6 \text { a } 84,6)\end{array}$ & \\
\hline 6 a 8 & $\begin{array}{c}79,5 \\
(75,7 a 82,8)\end{array}$ & & $\begin{array}{c}83,7 \\
(82,4 \text { a } 85,0)\end{array}$ & & $\begin{array}{c}83,4 \\
(82,2 \mathrm{a} 84,6)\end{array}$ & & $\begin{array}{c}83,9 \\
(82,6 \text { a } 85,0)\end{array}$ & & $\begin{array}{c}85,5 \\
(84,2 \mathrm{a} 86,6)\end{array}$ & & $\begin{array}{c}86,0 \\
(85,0 \text { a } 87,0)\end{array}$ & \\
\hline 9 a 11 & $\begin{array}{c}81,1 \\
(77,9 \text { a } 83,9)\end{array}$ & & $\begin{array}{c}82,8 \\
(81,4 \text { a } 84,2)\end{array}$ & & $\begin{array}{c}85,0 \\
(83,9 \text { a 86,1) }\end{array}$ & & $\begin{array}{c}84,7 \\
(83,4 \text { a } 85,9)\end{array}$ & & $\begin{array}{c}86,7 \\
(85,5 \text { a } 87,8)\end{array}$ & & $\begin{array}{c}85,3 \\
(84,2 \text { a } 86,4)\end{array}$ & \\
\hline \multicolumn{13}{|l|}{ Vive en hacinamiento } \\
\hline No & $\begin{array}{c}79,0 \\
(76,2 \text { a } 81,6)\end{array}$ & & $\begin{array}{c}81,6 \\
(80,4 \text { a } 82,7)\end{array}$ & & $\begin{array}{c}82,4 \\
(81,5 \text { a } 83,3)\end{array}$ & & $\begin{array}{c}83,3 \\
(82,3 \text { a } 84,2)\end{array}$ & $<0,001$ & $\begin{array}{c}85,1 \\
(84,2 \text { a 85,9) }\end{array}$ & $<0,001$ & $\begin{array}{c}84,5 \\
(83,7 \text { a 85,2) }\end{array}$ & 0,012 \\
\hline $\mathrm{Si}$ & $\begin{array}{c}79,1 \\
(75,1 \mathrm{a} 82,6) \\
\end{array}$ & 0,959 & $\begin{array}{c}78,6 \\
(76,8 \text { a } 80,4)\end{array}$ & 0,003 & $\begin{array}{c}81,7 \\
(80,1 \text { a } 83,1)\end{array}$ & 0,411 & $\begin{array}{c}80,2 \\
(78,6 \text { a } 81,7)\end{array}$ & & $\begin{array}{c}82,0 \\
(80,6 \text { a } 83,3)\end{array}$ & & $\begin{array}{c}82,6 \\
(81,4 \text { a } 83,8)\end{array}$ & \\
\hline
\end{tabular}

* Comparación con la proporción de cepillado 1 vez al día, mediante prueba Chi Cuadrado de Pearson.

** Diferencia establecida entre los estimadores porcentuales del año 2013 y 2018 mediante regresión logística binaria con link identity

*** Correlación establecida entre los años y el estimador porcentual de 2 a más cepillados por día para cada categoría de la característica evaluada.

† Coeficiente de correlación de Pearson.

‡ Coeficiente de correlación de Spearman-Brown.

Guión: cálculo de estimación no aplica. 
Tabla 3. Identificación multivariada de factores asociados a la adecuada frecuencia de cepillado dental en niños menores de 12 años. Perú 2013-2018

\begin{tabular}{|c|c|c|c|}
\hline Variables & OR & IC 95\% & Valor $p$ \\
\hline \multicolumn{4}{|l|}{ Año evaluado (Tendencia) } \\
\hline 2013 & \multicolumn{3}{|c|}{ Referencia } \\
\hline 2014 & 1,08 & 0,92 a 1,26 & 0,346 \\
\hline 2015 & 1,21 & 1,04 a 1,40 & 0,015 \\
\hline 2016 & 1,19 & 1,02 a 1,39 & 0,024 \\
\hline 2017 & 1,29 & 1,11 a 1,50 & 0,001 \\
\hline 2018 & 1,28 & 1,10 a 1,48 & 0,002 \\
\hline \multicolumn{4}{|l|}{$\begin{array}{l}\text { Alguna vez atendido en } \\
\text { odontología }\end{array}$} \\
\hline No & \multicolumn{3}{|c|}{ Referencia } \\
\hline Sí & 1,37 & 1,30 a 1,44 & $<0,001$ \\
\hline \multicolumn{4}{|l|}{$\begin{array}{l}\text { Recibió información sobre } \\
\text { higiene bucal }\end{array}$} \\
\hline No & \multicolumn{3}{|c|}{ Referencia } \\
\hline Sí & 1,38 & 1,31 a 1,45 & $<0,001$ \\
\hline No sabe / no recuerda & 0,96 & 0,69 a 1,33 & 0,813 \\
\hline Responde el mismo niño & 1,13 & 1,07 a 1,18 & $<0,001$ \\
\hline \multicolumn{4}{|l|}{ Comparte cepillo dental } \\
\hline Sí & \multicolumn{3}{|c|}{ Referencia } \\
\hline No & 2,30 & 1,46 a 3,60 & $<0,001$ \\
\hline \multicolumn{4}{|l|}{ Usa dentífrico para cepillarse } \\
\hline No usa & \multicolumn{3}{|c|}{ Referencia } \\
\hline Si usa & 1,72 & 1,49 a 1,99 & $<0,001$ \\
\hline \multicolumn{4}{|l|}{$\begin{array}{l}\text { Tiene algún tipo de seguro de } \\
\text { salud }\end{array}$} \\
\hline No & \multicolumn{3}{|c|}{ Referencia } \\
\hline Sí & 1,10 & 1,04 a 1,16 & 0,001 \\
\hline \multicolumn{4}{|l|}{ Región natural } \\
\hline Lima metropolitana & \multicolumn{3}{|c|}{ Referencia } \\
\hline Resto de la costa & 1,03 & 0,96 a 1,12 & 0,403 \\
\hline Sierra & 0,66 & 0,60 a 0,72 & $<0,001$ \\
\hline Selva & 0,93 & 0,84 a 1,02 & 0,101 \\
\hline \multicolumn{4}{|l|}{ Tipo de lugar de residencia } \\
\hline Urbano & \multicolumn{3}{|c|}{ Referencia } \\
\hline Rural & 0,88 & 0,81 a 0,95 & 0,001 \\
\hline \multicolumn{4}{|l|}{ Quintil de riqueza } \\
\hline Quintil inferior & 0,88 & 0,79 a 0,98 & 0,018 \\
\hline Segundo quintil & 0,73 & 0,66 a 0,80 & $<0,001$ \\
\hline Quintil intermedio & 0,72 & 0,66 a 0,79 & $<0,001$ \\
\hline Cuarto quintil & 0,80 & 0,73 a 0,87 & $<0,001$ \\
\hline Quintil superior & \multicolumn{3}{|c|}{ Referencia } \\
\hline \multicolumn{4}{|l|}{ Grupo etario (años) } \\
\hline 1 a 2 & \multicolumn{3}{|c|}{ Referencia } \\
\hline 3 a 5 & 1,58 & 1,49 a 1,68 & $<0,001$ \\
\hline 6 a 8 & 1,91 & 1,79 a 2,03 & $<0,001$ \\
\hline 9 a 11 & 1,96 & 1,82 a 2,10 & $<0,001$ \\
\hline
\end{tabular}

Modelo ajustado por características del hogar: Estructura del hogar, sexo de la cabeza del hogar, nivel educativo de la cabeza del hogar, cabeza del hogar estudiando en el año de evaluación, vive en hacinamiento.

los conglomerados, es algo que no se puede controlar en datos secundarios poblacionales, siendo esto una limitación importante que solicitamos se tenga en cuenta al referenciar estos estudios. Este hecho resalta la necesidad de estudios basales en toda la población, en los que se apliquen metodologías que puedan superar el sesgo de memoria que presenta la actual encuesta de salud bucal de la ENDES, en donde la información por auto-reporte se viene usando a pesar de su reconocido riesgo de sesgo.

Las limitaciones del presente trabajo recaen en la ausencia de una evaluación intraoral de los individuos que participaron de la encuesta que permita valorar la presencia de caries dental o valores de índice de higiene oral. En estas investigaciones se encuesta a los padres y sin evaluar su habilidad para realizar la higiene de sus hijos, las actitudes de los padres sobre la importancia del cepillado dental tiene una correlación directa sobre la calidad de vida ${ }^{(26)}$. Reportes previos concluyen que al concientizar a los padres mediante educación acerca de la importancia del cepillado y las visitas al odontólogo se incrementa la AFC y las visitas al consultorio dental ${ }^{(8,31)}$. El compartir el cepillo dental, ha sido relacionado a la disminución en la adherencia y tendencias de AFC, algo que podría considerarse un inherente al hecho de vivir en situación de pobreza y no contar con los medios para adquirir un cepillo para cada miembro de la familia.

La promoción de la salud mejora la calidad de vida pero requiere un compromiso en aras de poner práctica conductas más saludables, esto sólo se puede lograr cuando la promoción de la salud oral se implementa a nivel comunitario incorporando a varios actores (32). Estudios recientes han demostrado que la educación desde edades tempranas tiene un impacto en la creación de estas conductas protectoras ${ }^{(8,10)}$. Una estrategia puesta en práctica en Nueva Zelanda ${ }^{(33)}$ utilizando recordatorios en mensajes de texto (SMS) para una AFC demostró promover su incremento de manera significativa, lo que podría convertirse en una alternativa viable para comunidades alejadas o centros poblados en los que no se encuentre un servicio sanitario cercano.

En Perú, el programa de Presupuesto por Resultados (PpR) establece iniciativas gubernamentales en base a los resultados en la población a través de los bienes y servicios necesarios para lograrlos, que institucionaliza una política de rendición de cuentas por parte de los responsables o gestores de los proyectos que los conmina a presentar los resultados logrados y de esta forma se pueda medir la costo-efectividad de las medidas implementadas (34). Esta modalidad presupone la definición de resultados y productos desde la fase de proyecto (bienes y servicios), y los costos que requieren para su implementación, lo segundo un cambio significativo en la disponibilidad de la información, el manejo de esta por los tomadores de decisiones para garantizar la transparencia en la gestión pública. Bajo la figura de la educación en salud bucal y la implementación de un programa de concientización sobre la importancia del cepillado y el uso de un cepillo por persona, involucrando actores comunitarios y posibles medidas tecnológicas como recordatorios por mensaje de texto, se podría reducir la carga de las enfermedades orales de una manera efectiva y económica, mejorando 
de este modo la calidad de vida de las poblaciones más vulnerables. El hecho de que se hayan demostrado asociaciones positivas con la disponibilidad de servicios sanitarios debe tomarse como un estímulo para expandir dichos alcances a un mayor porcentaje de individuos, para así incrementar la AFC en la población peruana.

La presente investigación concluye que el porcentaje de AFC ha aumentado en los últimos seis años, siendo influenciado favorablemente por los aspectos sanitarios; a nivel rural este porcentaje es significativamente menor, algo que también se evidencia en la región sierra. Proyectos de extensión de los servicios sanitarios pueden ser una alternativa para incrementar los porcentajes de adherencia a una práctica de AFC en poblaciones vulnerables en zonas alejadas a donde se presenten servicios sanitarios y se pueda reducir el riesgo a caries dental y enfermedad periodontal desde edades tempranas.

Contribución de los autores: GS ha participado en la concepción y diseño del artículo, recolección de los datos. GS y WM participaron en el análisis estadístico de datos. Todos los autores participaron en la interpretación de los datos, redacción del manuscrito, revisión crítica del manuscrito y aprobaron la versión final.

Fuentes de financiamiento: autofinanciado.

Conflictos de interés: los autores declaran no tener conflictos de interés.

Material suplementario: disponible en la versión electrónica de la RPMESP.

\section{REFERENCIAS BIBLIOGRÁFICAS}

1. Petersen PE. The World Oral Health Report 2003: Continuous improvement of oral health in the 21 st century - The approach of the WHO Global Oral Health Programme. Community Dent Oral Epidemiol. 2003;31(Suppl. 1):S324. doi: 10.1046/j..2003.com122.x

2. Van Dyke TE, Sheilesh D. Risk factors for periodontitis. J Int Acad Periodontol. 2005;7(1):3-7. PMCID: PMC1351013

3. Pitts NB, Zero DT, Marsh PD, Ekstrand K, Weintraub JA, Ramos-Gomez F, et al. Dental caries. Nat Rev Dis Prim. 2017;3:1-16. doi: 10.1038/nrdp.2017.30

4. Kassebaum NJ, Bernabé E, Dahiya M, Bhandari B, Murray CJL, Marcenes W. Global burden of untreated caries: A systematic review and metaregression. J Dent Res. 2015;94(5):650-8. doi: $10.1177 / 0022034515573272$

5. Frencken JE, Sharma P, Stenhouse L, Green D, Laverty D, Dietrich T. Global epidemiology of dental caries and severe periodontitis - a comprehensive review. J Clin Periodontol. 2017;44(Suppl. 18):S94-105. doi: 10.1111/jcpe.12677

6. John MT, Hujoel P, Miglioretti DL, LeResche L, Koepsell TD, Micheelis W. Dimensions of oral-health-related quality of life. J Dent Res. 2004;83(12):956-60. doi: $10.1177 / 154405910408301213$

7. Loe H. Oral hygiene in the prevention of caries and periodontal disease. Int Dent J. 2000;50(3):129-39. doi: 10.1111/j.1875595x.2000.tb00553.x

8. Villena RS, Pesaressi E, Frencken JE. Reducing carious lesions during the first 4 years of life. J Am Dent Assoc. 2019; 150(12):1004-14. doi: 10.1016/j. adaj.2019.04.003
9. Melo P, Marques S, Silva OM. Portuguese self-reported oral-hygiene habits and oral status. Int Dent J. 2017;67(3):139-47. doi: $10.1111 /$ idj.12273

10. Harris N, García-Godoy F. Primary preventive dentistry. 6ta ed. New Jersey: Pearson Prentice Hall; 2004.

11. Ismail AI, Tellez M, Pitts NB, Ekstrand KR, Ricketts D, Longbottom C, et al. Caries management pathways preserve dental tissues and promote oral health. Community Dent Oral Epidemiol. 2013;41(1):e12-40. doi: 10.1111/ cdoe.12024

12. Lertpimonchai A, Rattanasiri S, Arj-Ong Vallibhakara S, Attia J, Thakkinstian A. The association between oral hygiene and periodontitis: a systematic review and meta-analysis. Int Dent J.2017;67(6):33243. doi: 10.1111/idj.12317

13. Van DerMaarel-WierinkCD, Vanobbergen JNO, Bronkhorst EM, Schols JMGA, De Baat C. Oral health care and aspiration pneumonia in frail older people: A systematic literature review. Gerodontology. 2013;30(1):3-9. doi: 10.1111/j.17412358.2012.00637.x

14. De Oliveira C, Watt R, Hamer M. Toothbrushing, inflammation, and risk of cardiovascular disease: Results from Scottish Health Survey. BMJ. 2010. doi: 10.1136/bmj.c2451

15. Li Q, Chalmers J, Czernichow S, Neal B, Taylor BA, Zoungas S, et al. Oral disease and subsequent cardiovascular disease in people with type 2 diabetes: a prospective cohort study based on the Action in Diabetes and Vascular Disease: Preterax and Diamicron Modified-Release Controlled Evaluation (ADVANCE) trial. Diabetologia. 2010;53(11):2320-7. doi: 10.1007/s00125-010-1862-1

16. Listl S, Galloway J, Mossey PA, Marcenes W. Global economic impact of dental diseases. J Dent Res. 2015;94(10):135561. doi: 10.1177/0022034515602879

17. Moriarty F, Hardy C, Bennett K, Smith SM, Fahey T. Trends and interaction of polypharmacy and potentially inappropriate prescribing in primary care over 15 years in Ireland: A repeated crosssectional study. BMJ Open. 2015;5(9):17. doi: 10.1136/bmjopen-2015-008656

18. Gutiérrez C, Roque J, Romaní F, Zagaceta J. Pravelencia de sintomáticos respiratorios en población peruana de 15 a más años: análisis secundario de la encuesta demográfica y de salud familiar, 2013 2015. Rev Peru Med Exp Salud Publica. 2017;34(1):98-104. doi: 10.17843/ rpmesp.2017.341.2771

19. Hernández-Vásquez A, Chacón-Torrico H. Manipulación, análisis y visualización de datos en la encuesta demográfica y de salud familiar con el programa R. Rev Peru Med Exp Salud Publica. 2019;36(1):128 33. doi: $10.17843 /$ rpmesp.2019.361.4062

20. Instituto Nacional de Estadística e Informática. Los adolescentes y su comportamiento reproductivo 2013 [Internet]. 1ra Ed. Lima: INEI; 2015. Disponible en: https://www.inei.gob.pe/ media/MenuRecursivo/publicaciones_ digitales/Est/Lib1199/libro.pdf

21. Lalithambigai G, Rao A, Rajesh G, Shenoy R, Pai M. Dental health behavior pertaining to fluoridated toothpaste usage among the parents of private and government school students in Mangalore, South India. J Indian Assoc Public Heal Dent. 2019;17(3):218-23. doi: 10.4103/ jiaphd.jiaphd_10_19 
22. Morón EM, Tomar S, Balzer J, Souza R. Hospital inpatient admissions for nontraumatic dental conditions among Florida adults, 2006 through 2016. J Am Dent Assoc. 2019;150(6):514-21. doi: 10.1016/j.adaj.2019.01.001

23. Tomar S, Carden D, Dodd V, Catalanotto F, Boylston J. Trends in dental-related use of hospital emergency departments in Florida. J Public Heal Dent. 2016;76(3):249-57. doi: $10.1111 /$ jphd.12158

24. Koivusilta L, Honkala S, Honkala E. Toothbrushing as a part of adolescent lifestyle predicts educational level. J Dent Res. 2003;82:361-6. doi: $10.1177 / 154405910308200507$

25. Jiménez R, Tapias-Ledesma MA, GallardoPino C, Carrasco P, De Miguel ÁG. Influence of sociodemographic variables on use of dental services, oral health and oral hygiene among Spanish children. Int Dent J. 2004;54(4):187-92. doi: 10.1111/j.1875595X.2004.tb00279.x

26. Paula JS, Leite ICG, Almeida AB, Ambrosano GMB, Pereira AC, Mialhe FL. The influence of oral health conditions, socioeconomic status and home environment factors on schoolchildren's self-perception of quality of life. Heal Qual Life Outcomes. 2012;10(6):1-8. doi: $10.1186 / 1477-7525-10-6$
27. Rebelo MAB, Rebelo Vieira JM, Pereira JV, Quadros LN, Vettore MV. Does oral health influence school performance and school attendance? A systematic review and meta-analysis. Int J Paediatr Dent. 2018:1-11. doi: 10.1111/ipd.12441

28. Vašíčková J, Hollein T, Sigmundová D, Honkala S, Pavelka J, Kalman M. Trends in children's toothbrushing in the Czech republic from 1994 to 2014: Results of the HBSC study. Cent Eur J Public Heal. Central European Journal of Public Health; 2017;25(Supp 1):S57-9. doi: 10.21101/cejph.a4951

29. Åstrøm AN, Samdal O. Time trends in oral health behaviors among Norwegian adolescents: 1985-97. Acta Odontol Scand. 2001;59(4):193-200. doi: 10.1080/00016350152509193

30. Holstein BE, Bast LS, Brixval CS, Damsgaard MT. Trends in Social Inequality in Tooth Brushing among Adolescents: 1991-2014. Caries Res. 2015;49(6):595-9. doi: $10.1159 / 000441196$

31. Shaghaghian S, Bahmani M, Amin M. Impact of malocclusion on oral healthrelated quality of life of preschool children. Int J Dent Hyg. 2015;13(3):192-8. doi: 10.1111/idh.12129
32. Fraihat N, Madae'en S, Bencze Z, Herczeg A, Varga O. Clinical effectiveness and costeffectiveness of oral-health promotion in dental caries prevention among children: Systematic review and meta-analysis. Int J Env Res Public Heal. 2019;16(15):1-33. doi: 10.3390/ijerph16152668

33. Schluter P, Lee M, Hamilton G, Coe G, Messer-Perkins H, Smith B. Keep on brushing: A longitudinal study of motivational text messaging in young adults aged 18-24 years receiving Work and Income Support. J Public Health Dent. 2015;75(2):118-25. doi: 10.1111/ jphd.12079

34. Dirección General de Presupuesto Público. Reporte de seguimiento de progreso: Indicadores de los programas presupuestales [Internet]. Lima: Ministerio de Economía y Finanzas; 2016. Disponible en: https:// www.mef.gob.pe/contenidos/presu_publ/ ppr/rpte_progreso2015_cap4.pdf

Correspondencia: Gilmer Solis Sánchez Dirección: Jr. Jirón Pastaza 780. Breña, Perú.

Teléfono: (+511) 993592470

Correoelectrónico: remlig@outlook.com 